\title{
钙钛矿组分和结构设计及其发光二极管器件性能研究进展
}

\author{
郭镇域周欢萍* \\ (北京大学工学院 北京 100871)
}

\begin{abstract}
摘要 有机-无机杂化鿭铁矿发光二极管(LED)的性能在短短几年时间内飞速提升, 近红外光器件的效率已达 $21.6 \%$, 绿光器件效率也达到 $20.3 \%$, 达到可以和商业化的有机发光二极管媲美的水平; 即使是稍有逊色的稳定性方面也有很 大进展, 报道的最长器件半衰期已达到 $250 \mathrm{~h}$. 器件性能的飞速提升得益于钙钛矿本身优异的光电性质, 而且通过丰富 的化学手段可进一步对锁铁矿材料的组分和结构进行调控, 从而优化器件性能. 本综述从组分设计、缺陷针化和界面 修饰的角度出发, 重点分析了组分和结构设计对钻铁矿 LED 器件效率和稳定性的影响, 最后对钻钑矿发光二极管的未 来发展进行展望.
\end{abstract}

关键词 钻铁矿; 发光二极管; 组分设计; 缺陷钝化; 界面修饰

\section{Research Progress of Composition and Structure Design in Perovskites for High Performance Light-emitting Diodes}

\author{
Zhenyu Guo Huanping Zhou* \\ (College of Engineering, Peking University, Beijing 100871, China)
}

\begin{abstract}
Over the past few years, the external quantum efficiency (EQE) of organic-inorganic hybrid perovskite light-emitting diodes (LEDs) has experienced a tremendous progress. The reported highest EQE of infrared and green perovskite LEDs has reached $21.6 \%$ and $20.3 \%$, respectively, which is comparable to commercial organic light-emitting diodes. Even the slightly inferior blue perovskite LEDs have a recorded EQE of $12.3 \%$. However, poor device stability is the biggest problem hindering the commercial application of perovskite optoelectronic devices, which is a severer problem in LEDs because the higher operating voltage will trigger acute ion migration. Encouragingly, however, the lifetime of perovskite LEDs has increased from the initial several seconds to over 200 hours, presenting a dramatic progress. The outstanding performance of perovskite LEDs is closely related with the excellent material properties, including tunable bandgaps, narrow full width at half maximum (FWHM), high defect tolerance, high electron/hole mobility and solution processibility. Moreover, the diversity of components and structure of perovskite makes the addition of various additives effective, enabling optimizing device performance through kinds of chemical methods. In this review, we analyzed the impacts of component and structure designing on the efficiency and stability of perovskite LEDs from the perspective of compositional designing, defect passivation and interfacial modification. We analyzed how compositional designing affects the optoelectronic properties through altering the ratio between organic and inorganic components or adding other molecules and ions to adjust the dimension of perovskites. As for defect passivation, we discussed various passivating reagents and their working mechanism. In addition, we summarized the significance of interfacial modification between different functional layers, including improving the wetting property of the substrates, achieving higher crystal quality of perovskite films, passivating the defects at the interfaces, blocking leakage current and reducing hole/electron injecting barrier. Finally, we discussed the scientific and technological challenges yet to be resolved before perovskite LEDs can be considered for commercial applications.
\end{abstract}

Keywords perovskite; light-emitting-diodes; compositional design; defect passivation; interfacial modification

\section{1 引言}

信息技术的快速发展造就了一个“信息时代” 的 21 世纪, 信息的显示、传输、处理、储存和识别技术的快 速发展使得新型信息材料和器件的研究变得火热. 信息 的显示作为其中重要一环, 近几十年来经历了快速更 迭, 从最简单的数码显示器, 发展到了阴极射线管显示 器、液晶显示屏和有机发光二极管(OLED)显示屏, 人们 对显示技术的体验和需求也因此达到了前所未有的高 度. 另外, 日益严重的能源危机使得显示和照明技术朝
着更加高效节能的方向发展, 传统低效率、高功耗的显 示和照明技术也逐渐被淘汰. 在显示器方面, 人们追求 的是高品质的显示图像(高分辨、高速度、宽视角、全 彩色)和便携的使用方式(轻薄、低功耗). 其中, OLED 由 于其轻薄、响应速度快、视角大、功耗低、色域广等特 点正逐渐替代传统的液晶显示屏和荧光灯, 成为一种新 型显示和照明技术.

虽然 OLED 有诸多优势, 并且其在智能手机、电视、 虚拟现实技术(VR)和可穿戴领域的市场前景一片大好,

\footnotetext{
*E-mail: happy_zhou@pku.edu.cn

Received October 9, 2020; published November 24, 2020.
} 
但是, 在 OLED 产业链的高端材料供应方面, 我国的面 板企业一直受制于人, 特别是 OLED 的核心发光材料, 其专利基本被国外厂商垄断. 苂光材料专利由出光兴 产、默克、LG、陶氏、德山、斗山等海外公司拥有，小 分子磷光 OLED 材料由美国 UDC 公司拥有, 热活化延 迟苂光材料(TADF)技术刚刚起步, 德日领先. 我国企业 只能以仿制技术含量较低的中间体和单体粗品为主. 因 此, 除了加大研发力度攻克有机发光材料的难点, 研发 新型发光材料也是一种发展策略.

有机-无机杂化钻钛矿是光伏领域的明星材料, 得 益于其优异的光电性质, 以钙钛矿作为吸光层的太阳能 电池在短短十年间, 效率从最初的 $3.8 \%$ 提升到了 $25.5 \%{ }^{[1-2]}$, 在效率上已经接近单晶硅太阳能电池. 除了 优异的光电性质, 钲钛矿可溶液加工以及带隙在可见光 到红外光范围可调的特性, 使得研究者们探索了其在其 它光电器件方面的应用, 比如发光二极管、光电探测器 和激光器等. 其实在 20 多年前, 就有研究者将二维铅卤 钲钛矿应用到发光二极管中, 但是当时器件只能在液氮 温度下发光, 限制了研究者们对钻钛矿 LED 的研究热 情 ${ }^{[3]}$. 直到近几年钙钛矿光伏的快速发展, 钙钛矿薄膜 突出的苂光量子产率和载流子迁移率, 使得钙钛矿再次 成为发光二极管发光层的很好选择.

自 2014 年首次在室温下点亮钙钛矿发光二极管以 来, 短短几年, 器件的外量子效率 (EQE) 从最初的 $0.76 \%$ (近红外光)、 $0.1 \%$ (绿光) ${ }^{[4]}$ 快速发展到了 $21.6 \%$ (近 红外光 $)^{[5]}$ 和 $20.3 \%$ (绿光 $)^{[6]}$, 难度较大的蓝光器件效率 也达到了 $12.3 \%{ }^{[7]}$. 仅从效率的角度来看, 钻钛矿 LED 器件的发展有很大的前景和希望, 但是稳定性一直是制 约钙钛矿光电器件发展的最大障碍, 目前钙钛矿 LED 器件最长的半衰期只有 $250 \mathrm{~h}^{[8]}$, 离商业化还有很远的 距离. 研究者们为了提高器件效率和稳定性做了大量的 工作. 本综述将从组分设计、缺陷针化和界面修饰的角 度出发, 重点分析钙钛矿组分和结构设计对钙钛矿 LED 器件效率和稳定性的影响, 并对钻钛矿 LED 未来 的发展方向进行展望.

\section{2 铅卤钲钛矿材料介绍}

钙钛矿得名于钻钛矿石中的钛酸钙 $\left(\mathrm{CaTiO}_{3}\right)$, 后来 泛指具有 $\mathrm{CaTiO}_{3}$ 晶体结构的化合物, 化学通式为 $\mathrm{ABX}_{3}$, 在当前研究火热的钙钛矿光电器件中, 钙钛矿材料的 $\mathrm{A}$ 位离子通常为有机阳离子或体积较大的金属阳离子, 如 $\mathrm{MA}^{+}\left(\mathrm{CH}_{3} \mathrm{NH}_{3}{ }^{+}\right), \mathrm{FA}^{+}\left(\mathrm{NH}_{2} \mathrm{CH}=\mathrm{NH}_{2}{ }^{+}\right), \mathrm{Cs}^{+}, \mathrm{Rb}^{+}$等; B 位 离子为二价的金属阳离子, 包括 $\mathrm{Pb}^{2+}, \mathrm{Sn}^{2+}$ 等; $\mathrm{X}$ 位离子 为卤素离子, 包括 $\mathrm{Cl}^{-}, \mathrm{Br}^{-}, \mathrm{I}^{-}$等. 铅卤钙钛矿作为一种 直接带隙半导体, 材料本身以及相应的光电器件具有巨 大的应用前景, 可应用于太阳能电池 ${ }^{[9-12] 、}$ 发光二极

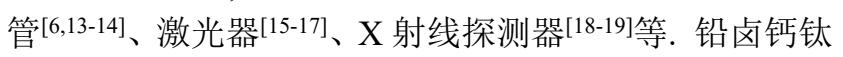
矿作为 LED 的发光层, 具有以下优势 ${ }^{[20]}$ : (1) 可通过组分
设计在可见光甚至近红外光范围调节发光峰位; (2)具有 较高的缺陷容忍度, 能够获得较高的苂光产率、发光纯 度和超宽的发光色域; (3)具有双极性电荷输运能力, 载 流子的迁移率较高, 器件能够获得较高的亮度; (4)钙钛 矿 LED 的制备成本低, 包括原材料、设备以及制备方法 都满足低成本的要求.

典型的钙钛矿 LED 器件是一种多功能层堆叠形成 的多层器件如图 1(a)所示, 钲钛矿发光层位于电子、空 穴传输层中间, 形成双异质结结构, 以限域注入的载流 子实现更好的发光 ${ }^{[21]}$. 其中, 钙钛矿层和部分传输层都 可以通过溶液旋涂的方法制备，这种低成本的制备方法 也是钻钛矿光电器件的优势. 空穴传输层通常是一种 $\mathrm{p}$ 型材料, 比如图 1(b)所示的聚(3,4-乙烯二氧噻吩)-聚(苯 乙烯磺酸酯)(PEDOT:PSS)、聚(双(4-苯基)(4-丁基苯基) 胺)(Poly-TPD)、氧化镍 $\left(\mathrm{NiO}_{x}\right)$ 等, 而电子传输层是一种 $\mathrm{n}$ 型材料, 比如图 1(b)所示的 1,3,5-三(1-苯基- $1 H$-苯并咪 唑-2-基)苯(TPBi)、4,7-二苯基-1,10-菲罗啉(Bphen)、氧 化锌/乙氧基聚乙烯亚胺 $(\mathrm{ZnO} / \mathrm{PEI})$ 等. 根据钙钛矿发光 层的价、导带边的位置选择功函数合适的电子、空穴传 输层, 以实现载流子的有效注入和辐射发光.
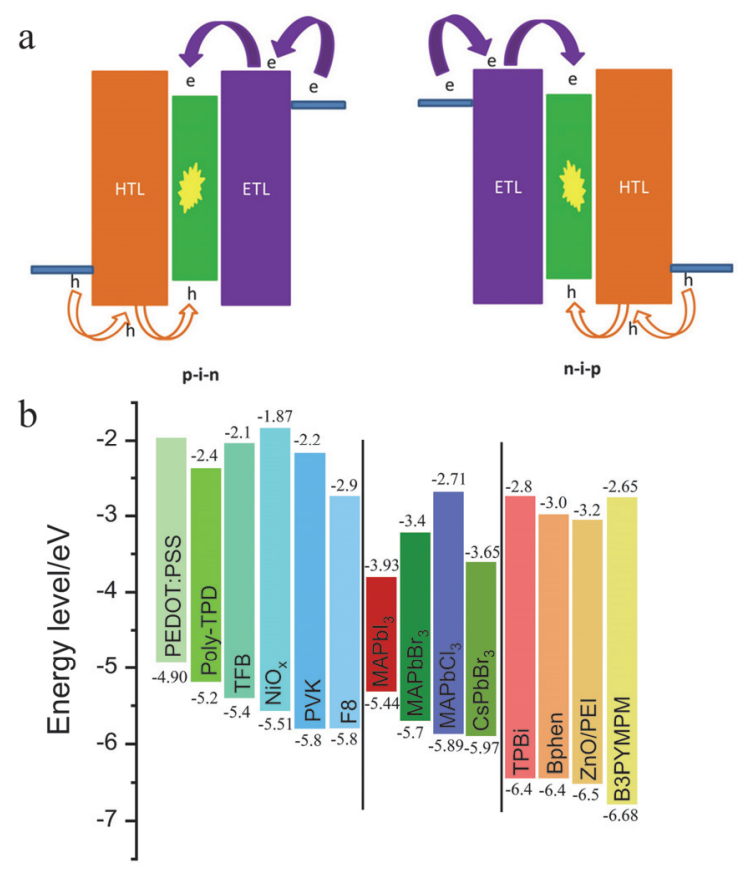

图 1 (a) 常见钻钛矿 LEDs 器件结构 ${ }^{[21]}$; (b) 不同类型的钻钛矿材料 和电子、空穴传输层材料的能级图 ${ }^{[21,23-26]}$

Figure 1 (a) Common perovskite LEDs device configurations ${ }^{[21]}$; (b) energy-level diagram of different kinds of perovskite and electron/hole transport layers ${ }^{[21,23-26]}$

LED 的基本工作原理是电致发光, 即电子-空穴或 激子在外电场作用下注入到发光层发生辐射复合发光. 其中, 外量子效率(EQE)是衡量发光二极管器件性能好 坏的重要参数, $\mathrm{EQE}$ 是指器件发射出来的光子数与注入 的电子空穴对的数量之比, 它的大小是器件内部多个物 
理过程的综合反映, 包括注入的电子和空穴形成电子空 穴对或激子的概率、电子空穴对或激子发生辐射复合的 概率以及辐射复合产生的光子从器件发射出来的概率 等. $\mathrm{EQE}$ 的大小与这些物理过程之间复杂的关系可以用 如下的公式表达 ${ }^{[22]}$.

$$
\mathrm{EQE}=f_{\text {balance }} \times \eta_{\mathrm{PLQY}} \times f_{\text {out-coupling }}
$$

其中, $f_{\text {balance }}$ 是载流子注入平衡系数, 主要与器件结构的 设计有关; $\eta_{\mathrm{PLQY}}$ 是材料的苂光量子产率(PLQY), 是材 料中辐射复合占总复合的比例; $f_{\text {out-coupling }}$ 是 LED 器件的 出光效率. 因此, 对 LED 器件性能的优化就主要从平衡 系数、PLQY 和出光效率这三方面着手, 在钙钛矿 LED 器件优化的过程中, 对钙钛矿材料组分和结构进行设计 的主要目的就是为了提高其 PLQY.

PLQY 是衡量一种发光材料性能好坏的重要参数, PLQY 的高低取决于材料在受激发后电子-空穴对或激 子发生辐射复合的速度和比例, 而材料中缺陷的存在不 利于其辐射发光, 为了改善材料的发光性能, 需要降低 材料中的缺陷密度. 钙钛矿有着较高的缺陷容忍度, 以 $\mathrm{MAPbI}_{3}$ 为例, 理论计算表明 [27], 如图 2 所示, 在 $\mathrm{MAPbI}_{3}$ 中, 缺陷形成能较低的几类缺陷比较容易形成, 包括填隙缺陷 $\left(\mathrm{I}_{\mathrm{i}}\right.$ 和 $\left.M A_{i}\right)$ 、空位缺陷 $\left(\mathrm{V}_{\mathrm{I}}, \mathrm{V}_{\mathrm{MA}}\right.$ 和 $\left.\mathrm{V}_{\mathrm{Pb}}\right)$ 、反 占位缺陷 $\left(\mathrm{MA} P \mathrm{~Pb}, \mathrm{~Pb}_{\mathrm{MA}}\right.$ 和 $\mathrm{MA}$ ), 但是这几类缺陷的能级 位置比较浅(距价、导带位置小于 $0.05 \mathrm{eV}$ ); 而一些深能 级缺陷的形成能又比较高, 不易形成, 包括铅填隙缺陷 $\left(\mathrm{Pb}_{\mathrm{i}}\right)$ 、反占位缺陷 $\left(\mathrm{I}_{\mathrm{MA}}, \mathrm{I}_{\mathrm{Pb}}\right.$ 和 $\left.\mathrm{Pb} \mathrm{b}_{\mathrm{I}}\right)$. 所以有机-无机杂化钙 钛矿中存在较多的是一些浅能级缺陷, 可以通过工艺优 化和后处理进行钝化, 获得缺陷少、苂光产率高的钙钛 矿材料. 较高的缺陷容忍度也是有机-无机钙钛矿材料 适合作为太阳能电池和发光二极管活性层的重要原因, 目前的大部分研究工作也都是为了减少钙钛矿中的缺 陷态.

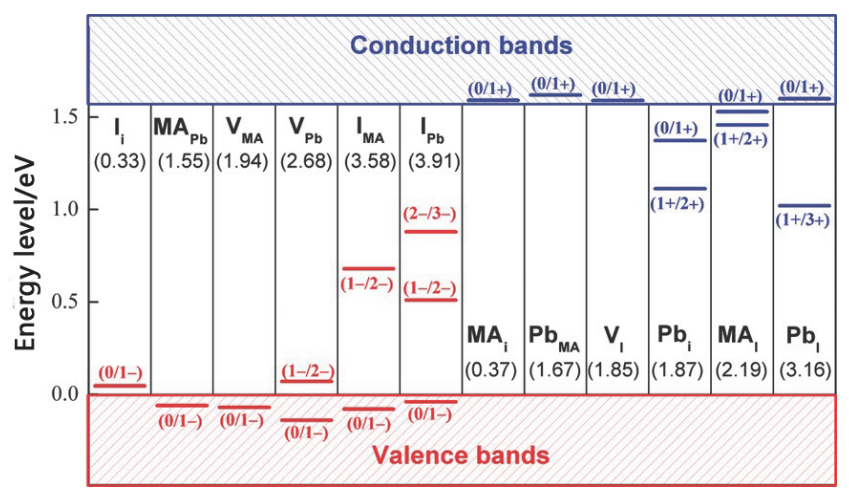

图 $2 \mathrm{MAPbI}_{3}$ 中各类点缺陷的计算能级位置和相应的形成能 ${ }^{[27]}$

Figure 2 Calculated transition energy levels and formation energies of point defects in $\mathrm{MAPbI}_{3}^{[27]}$

为了抑制电子-空穴被缺陷捕获，除了减少钙钛矿 中的缺陷态密度, 还可以通过抑制电子-空穴的迁移和 分离来实现, 将电子-空穴限域在一定空间内或者提高 钲钛矿的激子结合能也是提高苂光产率的方法. 在钙
钛矿量子点中, 通过特定的合成方法引入长链有机配 体，配体结合在钙钛矿晶粒表面限制了晶粒的长大，形 成了体积很小的钙钛矿纳米晶, 这种空间上的限域有效 地促进辐射复合的发生，纳米晶分散液的苂光产率能够 达到 $90 \%{ }^{[7,28]}$. 此外, 准二维钲钛矿体系中引入了长链 有机铵离子, 替代部分 $\mathrm{A}$ 位阳离子, 形成层状的准二维 钙钛矿. 准二维钙钛矿中存在空间限域效应和介电限域

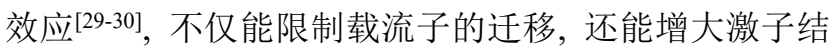
合能，提高辐射复合的速率，获得较高的苂光产率 ${ }^{[31]}$.

因此，通过钙钛矿的组分和结构设计，我们不仅可 以钝化钙钛矿中较多的浅能级缺陷，还可以对钙钛矿的 维度进行调控, 以期获得光电性质更加适合发光的材 料. 当然, 有机-无机钙钛矿作为 LED 的发光层还具备 其他优势: 载流子迁移率高, 溶液法制备, 制作成本低, 可柔性和大面积制备等 ${ }^{[32]}$.

\section{3 组分设计对钙钛矿 LED 器件性能的影响}

钙钛矿的组分设计是指改变钙钛矿中有机组分和 无机组分的比例或者加入组分构成之外的其他分子或 离子以形成新的维度和结构, 以获得更优的光电性能. 不同维度的钙钛矿体系的组分构成和特点差别较大, 比 如准二维体系中存在长链有机铵离子、量子点表面有大 量的有机配体，不同维度体系的组分设计方法和目的不 能一概而论. 因此, 我们将根据钙钛矿的不同维度, 来 对相应的组分设计方法进行讨论.

\section{1 三维钙钛矿 $\mathrm{ABX}_{3}$}

三维钙钛矿的组分设计对体系的缺陷密度和结晶 生长至关重要. 在三维钻钛矿 LED 器件中, 过量阳离子 是普遍采用的优化策略, 即 $\mathrm{AX}: \mathrm{PbX}_{2}$ 的投料物质的量比 大于 1 . Lee 等 ${ }^{[33]}$ 调节了 $\mathrm{MAPbBr}_{3}$ 前驱体中 $\mathrm{MABr}$ 和 $\mathrm{PbBr}_{2}$ 的比例, 发现当投料比从 $1: 1$ 提高到 $1.05: 1$ 时, 钙钛矿膜的苂光效率从 $3 \%$ 提高到 $36 \%$, 钙钛矿膜的平 均荧光寿命也从 $12.2 \mathrm{~ns}$ 提高到 $51.0 \mathrm{~ns}, \mathrm{LED}$ 器件的电流 效率从 $0.183 \mathrm{~cd} \cdot \mathrm{A}^{-1}$ 提高到 $42.9 \mathrm{~cd} \cdot \mathrm{A}^{-1}$, 外量子效率达 $8.53 \%$. 作者认为 $1: 1$ 投料时, 钻钛矿膜的晶界处存在 未配位的金属铅，导致严重的非辐射复合，而稍过量的 $\mathrm{MABr}$ 能提供足够的溴离子抑制未配位铅的形成，减少 缺陷, 防止载流子猝灭(如图 $3 \mathrm{a}$ 所示). 表 1 所示为文献 报道的三维钙钛矿 LED 器件的组分调控方案和性能参 数, 可以看到, 在不同的阳离子体系或卤素体系中, 研 究者们为了提高 LED 器件性能, 都会增大钙钛矿中 AX 组分的比例. Park 等 ${ }^{[34]}$ 在 $\mathrm{MAPbBr}_{3}$ 的体系中加入了过量 的 $\mathrm{MABr}$ ，他们认为过量的 $\mathrm{MABr}$ 有两个作用: (1)在 $\mathrm{MAPbBr}_{3}$ 前驱液中, $\mathrm{MABr}$ 可视为一种 “表面活性剂”, 将前驱液中形成的 $\mathrm{MABr} \cdot \mathrm{PbBr}_{2} \cdot 2 \mathrm{DMSO}$ 加合物孤立开, 最终在 $\mathrm{MABr}$ 基质中形成纳米尺寸的钙钛矿晶粒; (2)有 机盐 MABr 的带隙比钲钛矿宽, 能够形成 I 型能级排布 (图 3b), 将电子、空穴限域在纳米尺寸的钻钛矿晶粒中, 


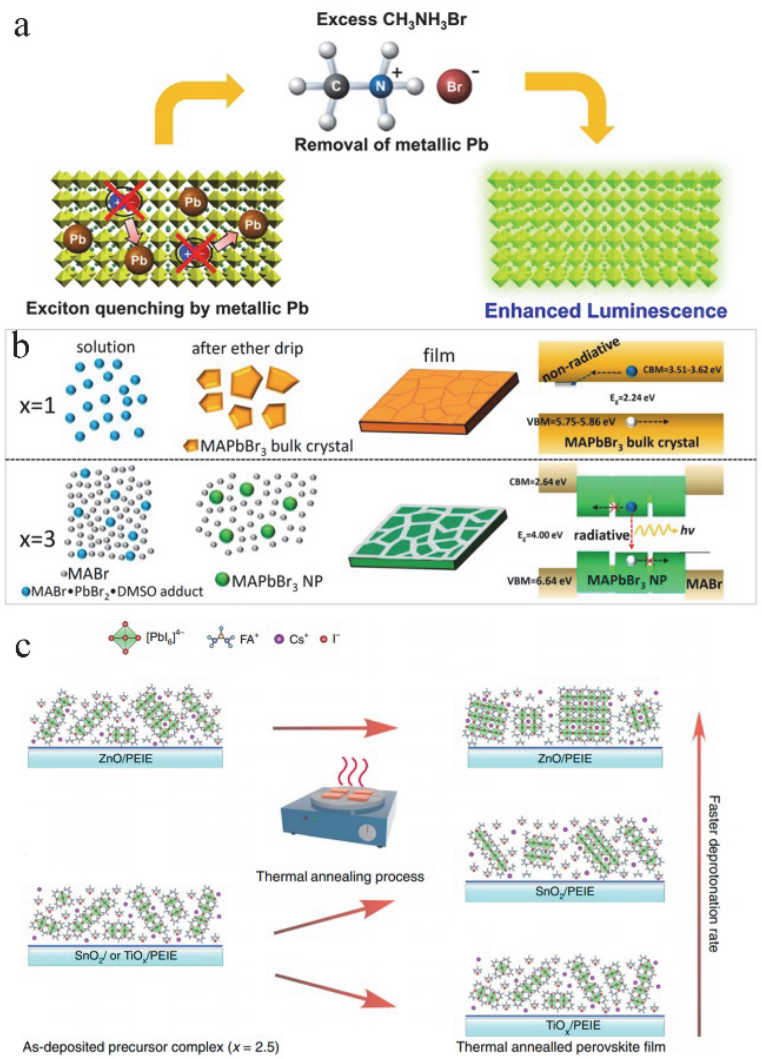

图 3 (a) 过量的 $\mathrm{MABr}$ 抑制金属 $\mathrm{Pb}$ 对激子的猝灭 ${ }^{[33]}$; (b) 不同 $\mathrm{MABr}$ 含量对钻钛矿结晶和载流子复合过程的影响 ${ }^{[34]}$; (c) 钙铁矿在不同基 底上的结晶过程 ${ }^{[36]}$

Figure 3 (a) Stoichiometry control using excess $\mathrm{MABr}$ to prevent exciton quenching from metallic $\mathrm{Pb}$ atoms ${ }^{[33]}$; (b) effect of different $\mathrm{MABr}$ content on the crystallization and carrier recombination process of perovskite $^{[34]}$; (c) crystallization of perovskite on different substrates ${ }^{[36]}$

表 1 三维钙钣矿 LED 器件中钙钣矿组分调控和性能参数

Table 1 Composition control and performance parameters in three-dimensional perovskite LED devices

\begin{tabular}{|c|c|c|c|}
\hline $\begin{array}{c}\begin{array}{c}\text { 钻钛矿 } \\
\text { 组分 }\end{array} \\
\end{array}$ & 最佳物质的量比 & LED 器件性能 & $\begin{array}{l}\text { 参考 } \\
\text { 文献 }\end{array}$ \\
\hline $\mathrm{CsPBr}_{3}$ & $\mathrm{CsBr} / \mathrm{PbBr}_{2}=2$ & $\begin{aligned} L_{\max } & =407 \mathrm{~cd} \cdot \mathrm{m}^{-2} \\
\mathrm{CE}_{\max } & =0.035 \mathrm{~cd} \cdot \mathrm{A}^{-1}\end{aligned}$ & [38] \\
\hline $\mathrm{CsPbBr}_{3}$ & $\mathrm{CsBr} / \mathrm{PbBr}_{2}=1.5$ & $\begin{array}{l}L_{\max }=23828 \mathrm{~cd} \cdot \mathrm{m}^{-2} \\
\mathrm{CE}_{\max }=9.54 \mathrm{~cd} \cdot \mathrm{A}^{-1}\end{array}$ & [39] \\
\hline $\mathrm{CsPBrr}_{3}$ & $\mathrm{CsBr} / \mathrm{PbBr}_{2}=1.1$ & $\begin{array}{l}L_{\max }=13752 \mathrm{~cd} \cdot \mathrm{m}^{-2} \\
\mathrm{CE}_{\max }=5.39 \mathrm{~cd} \cdot \mathrm{A}^{-1}\end{array}$ & [37] \\
\hline $\mathrm{FAPbBr}_{3}$ & $\mathrm{FABr} / \mathrm{PbBr}_{2}=2$ & $\begin{array}{c}L_{\max }=109000 \mathrm{~cd} \cdot \mathrm{m}^{-2} \\
\mathrm{CE}_{\max }=21.3 \mathrm{~cd} \cdot \mathrm{A}^{-1} \\
\mathrm{EQE}_{\max }=4.66 \%\end{array}$ & [40] \\
\hline $\mathrm{MAPbBr}_{3}$ & $\mathrm{MABr} / \mathrm{PbBr}_{2}=3$ & $\begin{array}{c}\mathrm{CE}_{\max }=34.46 \mathrm{~cd} \cdot \mathrm{A}^{-2} \\
\mathrm{EQE}_{\max }=8.21 \%\end{array}$ & [34] \\
\hline $\mathrm{FAPbI}_{3}$ & $\mathrm{FAI} / \mathrm{PbI}_{2}=2.4$ & $\begin{array}{c}\mathrm{EQE}_{\max }=20.7 \% \\
\mathrm{ECE} @ 100 \mathrm{~mA} \cdot \mathrm{cm}^{-2}= \\
12 \%{ }^{a}\end{array}$ & {$[14]$} \\
\hline $\begin{array}{c}\mathrm{FAPbI}_{3} \\
(\mathrm{FACs}) \mathrm{PbI}_{3}\end{array}$ & $\begin{array}{c}\mathrm{FAI} / \mathrm{PbI}_{2}=2 \\
(\mathrm{FACs}) \mathrm{I} / \mathrm{PbI}_{2}=2.5\end{array}$ & $\begin{array}{c}\mathrm{EQE}_{\max }=14.2 \% \\
\mathrm{CE}_{\max }=10.7 \% \\
\mathrm{EQE}_{\max }=19.6 \%\end{array}$ & [41] \\
\hline
\end{tabular}

$a$ 能量转换效率(ECE).
促进了辐射复合的发生. Zhao 等 ${ }^{[35]}$ 在 $\mathrm{Cs}_{0.17} \mathrm{FA}_{0.83} \mathrm{PbI}_{2.5}$ $\mathrm{Br}_{0.5}$ 体系中研究了过量 FAI 对钙铁矿薄膜的结晶过程以 及 LED 器件性能的影响. 他们通过原位表征发现过量 的FAI会促使钙钛矿在成膜过程中先形成宽带隙的中间 相，在退火后形成结晶度更高、取向更集中的钙钛矿薄 膜，并且过量的 FAI 会聚集在晶体的表、界面，针化晶 界的缺陷. 制备的器件 EQE 从 $0.25 \%$ 提高到 $17.4 \%$. Gao 等[36]发现在 $\mathrm{FAPbI}_{3}$ 体系中，虽然提高FAI 的比例能够针 化钙钠矿中的缺陷，提高钙钛矿膜的苂光寿命和 PLQY, 但是过量的 FAI 会影响钻铁矿的结晶, 在成膜过程中会 “包裹” $\left[\mathrm{PbI}_{6}\right]^{4-}$ 结构单元, 形成大量的低维中间相, 阻碍 三维钙铁矿结构的形成. 他们对比了三种不同的基底 $\left(\mathrm{ZnO} 、 \mathrm{TiO}_{2} 、 \mathrm{SnO}_{2}\right)$, 发现只有 $\mathrm{ZnO}$ 基底上能形成高质 量的钙钛矿膜. 这是因为表面更偏碱性的 $\mathrm{ZnO}$ 基底在 钙钛矿成膜过程中对 $\mathrm{FA}^{+}$离子有去质子作用, 从而消除 过量 FAI 抑制钙钛矿结晶的效应. 因此, 只有过量 FAI 与碱性基底 $\mathrm{ZnO}$ 协同发挥作用, 才能获得强发光的三 维钙铁矿 (图 3c). Lee 等 ${ }^{[37]}$ 通过 X 射线光电子能谱(XPS) 证明过量 $\mathrm{CsBr}$ 能抑制 $\mathrm{CsPbBr}_{3}$ 中金属 $\mathrm{Pb}$ 的形成, 消除 未配位铅形成的缺陷态. 但是器件的正、反扫描电流电压数据显示, 过量的 $\mathrm{CsBr}$ 会导致更严重的迟滞现象, 作者认为是 $\mathrm{Br}$ 离子的迁移更加容易导致了更明显的迟 滞现象.

综上所述, 在三维钙铁矿体系中 $\mathrm{AX}$ 的含量至关重 要, 适量增加 $\mathrm{AX}$ 的比例能提供富卤素的环境, 减少卤 素离子空位，抑制金属 $\mathrm{Pb}$ 的形成; 进一步增加 $\mathrm{AX}$ 的比 例，则会影响钙钛矿的成核结晶过程，适当的比例能减 小钙钛矿晶粒尺寸，促进辐射复合的发生，但是太过量 的 $\mathrm{AX}$ 不利于钻铁矿的晶体生长, 影响结晶质量. 为了 获得发光性能最优的䥻钛矿体系, 需要对组分比例、基 底性质和制备工艺等因素进行综合调控. 此外, 卤素盐 中阳离子的半径、离子键的强弱也会影响钙钛矿薄膜的 性质，比如，卤素盐中阳离子半径决定了其能否进入钲 铁矿晶格, 从而对钻钣矿的结晶生长产生不同的影响; 卤素盐的离子键强度对后续薄膜中卤素的迁移也会有 影响, 该内容会在第 4 节详细讨论.

\section{2 准二维钙钛矿 $\mathrm{L}_{2}\left(A B X_{3}\right)_{n-1} \mathrm{BX}_{4}$}

准二维钙钛矿中长链铵离子的引入改变了钙钛矿 的结构和维度, 赋予准二维钙铁矿体系独特的光电性 能, 如: 天然的量子阱结构、能量转移机制等. 准二维钙 铁矿的组分设计通过改变体系的结构类型、相分布来影 响材料的光电性质, 从而影响最终器件的性能表现.

首先, 准二维钙钛矿与三维钲铁矿在结构上存在很 大差异, 结构的差异赋予其独特的光电性能. 准二维钙 钣矿中引入的长链铵离子半径较大, 无法进入共角连接 的铅卤八面体形成的无机框架中, 将三维钙钛矿分割成 片层状的准二维结构, 如图 4a 所示, 有机相形成的中间 插层与钙铁矿无机相交替排布, 其中无机层的层数定义 
为 $n$ 值, $n$ 值可以通过大阳离子 $\left(\mathrm{L}^{+}\right)$和小阳离子 $\left(\mathrm{A}^{+}\right)$的比 例来调控 ${ }^{[42]}$. 有机、无机相的交替排布使得准二维钻钛 矿存在空间限域和介电限域效应 ${ }^{[29-30]}$. 其中, 空间限域 效应是指, 有机铵的插层能够在准二维钙钛矿中形成天 然的量子阱结构, 并且无机层厚度越薄 $(n$ 值越小), 对应 的准二维钙钛矿的带隙越宽, 因此可通过调节大、小阳 离子的比例来改变准二维钻钛矿的发射波长; 介电限域 效应是指有机、无机层差异较大的介电常数使得准二维 钻钣矿的激子结合能增大, 有利于辐射复合的发生 ${ }^{[31]}$. 因此, 限域效应的存在是准二维钻钛矿作为发光材料的 优势之一. 此外, 由于有机相和无机相结晶速率的差 异 ${ }^{[43]}$, 准二维䥻钛矿薄膜不是单一 $n$ 值的纯相, 而是 $n$ $=1,2,3, \ldots, \infty$ 的相并存的混相结构. 在受到激发之后, 不同 $n$ 值的相之间发生快速的能量转移, 载流子会集中 到带隙较窄的大 $n$ 值的区域, 如图 $4 \mathrm{~b}$ 所示, 所以准二维 钙铁矿的混相结构可看做是 “载流子的集中器” (carrier concentrators), 增大了大 $n$ 值区域的载流子密度, 更有 利于辐射复合的发生 ${ }^{[29,31,44]}$. 复杂的准二维钙钛矿体系 使得组分的设计更具多样性, 本小节重点论述大阳离 子、小阳离子的种类和比例对准二维钻钣矿的结构类 型、维度调控和相分布的影响.

a

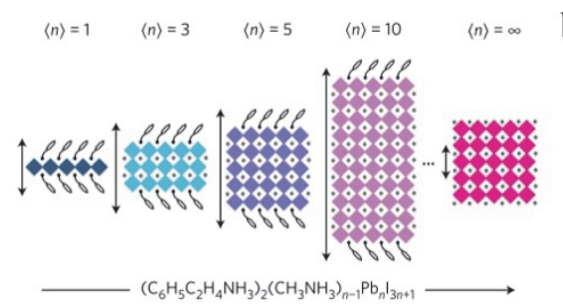

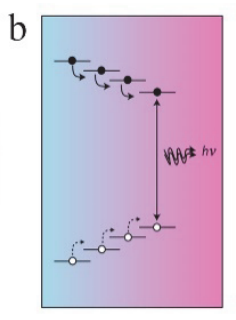

图 4 (a) 准二维钻钛矿中不同 $n$ 值相的结构单元, 表示从二维 $(n=1)$ 到三维 $(n=\infty)$ 的变化; (b) 多相钻钛矿在不均匀能级分布中的能量传 输过程, 将载流子集中到带隙最小的发光相. 箭头代表载流子的转移 过程 ${ }^{[29]}$

Figure 4 (a) Unit cell structure of Quasi-2D perovskites with different $\langle n\rangle$ values, showing the evolution of dimensionality from $2 \mathrm{D}(n=1)$ to 3D $(n=\infty)$; (b) multi-phase perovskite materials channel energy across an inhomogeneous energy landscape, concentrating carriers to smallest bandgap emitters. The arrows represent the carrier transfer process ${ }^{[29]}$

\subsection{1 小阳离子}

相较大阳离子, 小阳离子的选择较少, 限于 $\mathrm{MA}^{+}$、

$\mathrm{FA}^{+} 、 \mathrm{Cs}^{+} 、 \mathrm{Rb}^{+}$等, 小阳离子主要影响的是准二维钻钛 矿的生长动力学 ${ }^{[45]}$.

准二维钙钛矿生长动力学的变化会改变体系中不 同 $n$ 值的相分布, 影响不同相之间的能量转移, 从而影 响 LED 器件的发光性能. You 等 ${ }^{[25]}$ 首先在 $\mathrm{PEA}_{2}\left(\mathrm{FAPbBr}_{3}\right)_{2} \mathrm{PbBr}_{4}$ 的准二维体系中, 通过组分调控 和表面缺陷钝化, 获得了发光峰在 $532 \mathrm{~nm}$, 外量子效率 为 $14.36 \%$ 的绿光 LED 器件. 他们发现, 在小阳离子为 $\mathrm{FA}$ 的体系中, 不同 $n$ 值的投料比获得的准二维钙钛矿 薄膜中的相分布有很大差异, 只有按照 $n=3$ 进行投料, 体系中小 $n$ 值相 $(<n>=2)$ 与大 $n$ 值的发光相的含量是相
对平衡的，才能获得更高的荧光效率(57.3\%)(图 5a). 另 外, You 等 ${ }^{[46]}$ 还比较了不同小阳离子对准二维钙钛矿 LED 性能的影响, 虽然同样工艺条件下 MA 和 FA 体系 的准二维钙钠矿薄膜的苂光效率相差不大(MA： $85.3 \%$; FA: 73.5\%), 但是相应的 LED 器件的外量子效率差别明 显(MA: 0.93\%; FA: 15.4\%). 他们发现, FA 体系中存在 较多的 $n=2$ 的相, 而 MA 体系中存在较多的 $n=1$ 的相 (图 5b). 虽然 $n=1$ 的相带隙更宽, 对载流子能起到更好 的限域作用, 但是 $n=1$ 的相较深的价带导致与空穴传 输层 PEDOT:PSS 之间的注入势垒更大, 空穴难以注入, 电子和空穴的注入不平衡, 导致了 MA 体系器件性能很 低(图 5c). Wang 等 ${ }^{[47-48]}$ 通过增大体系中小阳离子的比例 有效地抑制了准二维钻针矿 LED 器件在高激发强度下 发生效率滚降的问题. 他们认为, 效率滚降是由于在高 电压激发下, 载流子密度较高, 发生俄歇复合, 从而降 低了器件效率, 并且能量转移效应使得这一效应在准二 维钻铁矿中更加严重. 在量子阱结构中, 可以通过增大 量子阱的宽度来降低载流子密度, 抑制俄歇复合. 而准 二维钙钠矿中量子阱的宽度其实对应的是 $n$ 值的大小, 在增大小阳离子的比例时, 就能获得 $n$ 值增大的准二维 钙铁矿, 从而缓解俄歇复合引起的效率滚降问题. Sargent 等 ${ }^{[44]}$ 发现, 通过优化制备工艺和投料比(同时降低 大、小阳离子的比例), 能够形成 “分级的相分布” ，提 高能量转移的速度和效率, 使得载流子被缺陷捕获前转 移到大 $n$ 值相进行辐射复合, 提高了器件的效率.
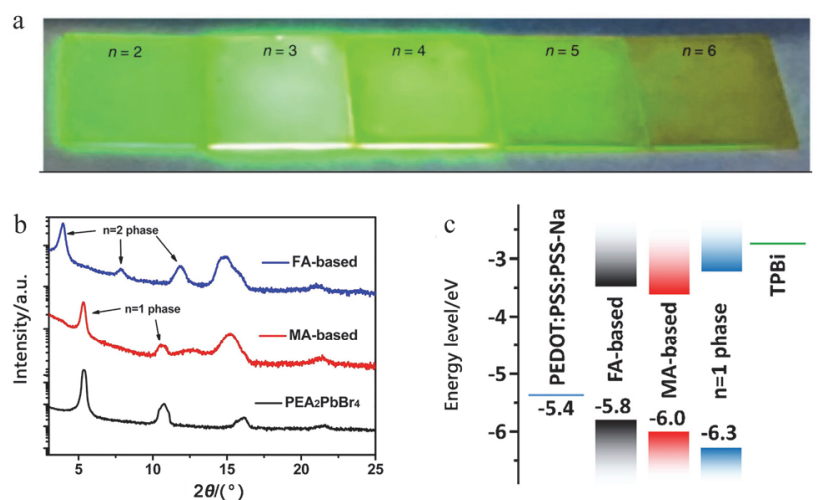

图 5 (a)不同 $n$ 值的准二维钙钛矿薄膜 $\left(\mathrm{PEA}_{2}\left(\mathrm{FAPbBr}_{3}\right)_{n-1} \mathrm{PbBr}_{4}\right)$ 在紫 外灯照射下的苂光图片 ${ }^{[25]}$; (b) $n=1$ 的二维钲钛矿 $\left(\mathrm{PEA}_{2} \mathrm{PbBr}_{4}\right)$ 薄膜、 $\mathrm{MA}$ 基 $\left(\mathrm{PEA}_{2}\left(\mathrm{MAPbBr}_{3}\right)_{2} \mathrm{PbBr}_{4}\right) 、 \mathrm{FA}$ 基 $\left(\mathrm{PEA}_{2}\left(\mathrm{FAPbBr}_{3}\right)_{2} \mathrm{PbBr}_{4}\right)$ 的准二 维钻钛矿薄膜的 X 射线衍射(XRD)图; (c) $n=1$ 的相、MA 基、 FA 基 的准二维钻钛矿与载流子传输层的能级排布 ${ }^{[46]}$

Figure 5 (a) Photoluminescence image of $\mathrm{PEA}_{2}\left(\mathrm{FAPbBr}_{3}\right)_{n-1} \mathrm{PbBr}_{4}$ films with different compositions under ultraviolet lamp excitation ${ }^{[25]}$; (b) X-ray diffraction (XRD) patterns of the $n=1$ phase film $\left(\mathrm{PEA}_{2} \mathrm{PbBr}_{4}\right)$, the MA-based $\mathrm{PEA}_{2}\left(\mathrm{MAPbBr}_{3}\right)_{2} \mathrm{PbBr}_{4}$ film, and the FA-based $\mathrm{PEA}_{2}\left(\mathrm{FAPbBr}_{3}\right)_{2} \mathrm{PbBr}_{4}$ film; (c) band alignment of the $n=1$ phase, MA-based and FA-based perovskites compared with the hole and electron injection layer materials ${ }^{[46]}$

相分布的变化除了对发光效率有影响, 还会改变体 系的发射峰位, 所以在制备红、蓝光 LED 器件时也需要 考虑小阳离子的影响. 虽然调节卤素的比例可以很容易 
地改变钙钛矿的带隙, 但是采用混合卤素方式获得红光 (碘/溴混合)和蓝光(溴/氯混合)的体系在电场作用下容 易发生相分离, 色稳定性差 ${ }^{[49]}$. 能量转移机制使得准二 维钙钛矿中的发射峰取决于体系中最大 $n$ 值的相, 而空 间限域效应能够使纯溴或纯碘体系的发射峰蓝移，所以 在准二维体系中只需要控制最大 $n$ 值相的发射峰就能获 得想要的蓝、红光体系. 但是研究表明 $[43,45]$, 大阳离子 之间较强的范德华作用使得 $n=1$ 的相最容易形成, 这 一趋势使得前驱体中剩余的组分倾向于形成大 $n$ 值的 相，这种明显的相偏析不利于控制体系的最大 $n$ 值. 优 化小阳离子的选择能够部分解决相偏析的问题, 目前大 部分红、蓝光的体系都采用体积较小的 $\mathrm{Cs}^{+}$离子作为小 阳离子 ${ }^{[23,43,50-53]}$. Mathews 等 ${ }^{[53]}$ 认为, 铯盐在有机溶剂中 溶解度相较于 MA、FA 等有机盐低, 所以在钙钛矿结晶 过程中较早达到饱和而参与准二维钙钛矿的生长, 并且 $\mathrm{Cs}^{+}$离子体积较小, 更容易进入低维钙钛矿的框架中, 所以体系中 $n=1$ 的低 $n$ 相含量更少, 减轻了准二维钙钛 矿中相偏析的程度.

另外, 小阳离子的选择可以直接改变铅卤键的构 型, 影响钻钛矿价、导带边的位置, 改变体系的发射峰. Chen 等 [50]首先制备了 $\mathrm{Cs}-\mathrm{Rb}$ 混相的三维钙钛矿 $(\mathrm{CsRb}) \mathrm{PbBr}_{3}, \mathrm{Rb}^{+}$的掺入将带隙从 $2.31 \mathrm{eV}\left(\mathrm{CsPbBr}_{3}\right)$ 增 大到 $2.48 \mathrm{eV}$ (发射峰 $499 \mathrm{~nm}$ ), 在引入苯乙铵形成准二 维钙钛矿后, 发射峰蓝移到 $466 \mathrm{~nm}$, 获得了色光较纯, 苂光产率高的蓝光钙钛矿薄膜, PLQY 达到 $82 \%$, 相应 的 LED 器件 EQE 达到 $1.35 \%$. You 等 ${ }^{[54]}$ 在溴基的准二维 钲钛矿中引入乙铵阳离子, 乙铵能部分取代 $\mathrm{Cs}^{+}$离子, 减小 $\mathrm{Pb}-\mathrm{Br}$ 轨道耦合, 增大带隙, 将体系的发射峰从 508 $\mathrm{nm}$ 蓝移到 $466 \mathrm{~nm}$. 最终他们获得了发射峰在 $488 \mathrm{~nm}$, EQE 达 $12.1 \%$ 的天蓝光钙钛矿 LED.

小阳离子的选择对钙钛矿的稳定性也有很大影响, 钲钛矿的不稳定性一部分是源于体系中的有机组分, 常 用的 $\mathrm{MA}^{+}$和 $\mathrm{FA}^{+}$的结构特点导致了二者稳定性的差异. $\mathrm{MA}^{+}$中的 $\mathrm{C}-\mathrm{N}$ 键和 $\mathrm{N}-\mathrm{H}$ 键是极性较强的共价单键, 化学键键能较小, 易断裂; 而 $\mathrm{FA}^{+}$中的大 $\pi$ 键使正电荷 离域化, 结构更加稳定. 所以在钻钛矿的稳定性方面, MA 体系的钻钛矿不是好的选择, 选择更加稳定的 $\mathrm{FA}^{+}$ 或无机离子 $\mathrm{Cs}^{+}$作为阳离子的效果会更好.

综上所述, 在准二维钙钛矿器件的优化过程中, 对 小阳离子的考虑不仅需要从阳离子的稳定性、相应材料 的缺陷态密度等方面考虑, 还需要考虑准二维钙钛矿的 $n$ 值分布、晶体取向等方面, 并且后者是准二维钙钛矿 器件中更关键的问题. 小阳离子通过影响钙钛矿的生长 动力学来调节体系中的相分布, 改变了发光效率和发光 峰位; 并且, 小阳离子的大小会影响铅卤八面体的构型, 通过改变主相的带隙来影响发光峰位. 所以为了获得高 效的准二维钙钛矿 LED, 需要对小阳离子的种类和数 量进行精细调控.

\subsection{2 大阳离子}

在准二维钲钛矿中，大阳离子的选择更加丰富，大 阳离子的链长、共轭基团、官能团数等对钲钛矿的光电 性能、缺陷态、载流子迁移率和结构类型有重要影响. Adachi 等 ${ }^{[55]}$ 在制备 $\mathrm{R}_{2} \mathrm{FA}_{n-1} \mathrm{~Pb}_{n} \mathrm{Br}_{3 n+1}$ 准二维钙钛矿 LED 器件时发现, 以苯乙铵作为大阳离子的器件外量子效率 可达 $12.4 \%$, 而菜甲铵的器件只有 $3.4 \%$, 他们认为是不 同大阳离子三线态能级的差异导致了这一现象. $\left[\mathrm{PbBr}_{6}\right]^{4-}$ 无机层的两个三线态能级为 $2.99 \mathrm{eV}$, 苯乙铵的 最低激发三线态比无机层的高, 为 $3.3 \mathrm{eV}$, 所以苯乙铵 不会猝灭钙钛矿中的三线态激子; 而萗甲铵的最低激发 三线态为 $2.6 \mathrm{eV}$, 比 $\left[\mathrm{PbBr}_{6}\right]^{4^{-}}$无机层和准二维钻钛矿的 三线态激子的能级都低, 因此 $\left[\mathrm{PbBr}_{6}\right]^{4^{-}}$中的 Wannier 激 子和准二维钙钛矿中的三线态激子会转移到䒺甲铵的 三线态能级上, 不利于准二维钙钛矿中不同 $n$ 值的相之 间的能量转移(图 6a). Nie 等[56]在制备 $\mathrm{R}_{2} \mathrm{MA}_{n-1} \mathrm{~Pb}_{n} \mathrm{Br}_{3 n+1}$ 准二维钻钛矿 LED 器件时也发现, 大阳离子的选择对 器件性能有很大影响. 以带苯环的有机铵(苯乙铵)作为 大阳离子制备的 LED 器件性能比线性有机铵(丁铵)更 好. 他们认为大阳离子的选择会影响钻钛矿的光电性 能, 苯乙铵作为阳离子时, 钻钛矿在受到光激发后, 空 穴会局域在 $\mathrm{Br}$ 的 $4 \mathrm{p}$ 轨道上，限制其被缺陷捕获发生非 辐射复合，而丁铵体系中离域的空穴容易被缺陷捕获， 导致苂光效率低. Tan 等 ${ }^{[57]}$ 在制备锡基 $\mathrm{R}_{2} \mathrm{SnI}_{4}$ 纯二维钲 钛矿 LED 时对比了苯乙铵和噻吩乙铵的差别, 他们发 现大阳离子对纯二维钙钛矿的带隙和激子结合能有很 大影响. 主要原因是大阳离子形成的有机插层介电常数 的不同, 而带隙、激子结合能都与 $\left[\mathrm{SnI}_{6}\right]^{4^{-}}$无机层和有机 层的介电常数之比有关, 所以大阳离子的选择对二维钙
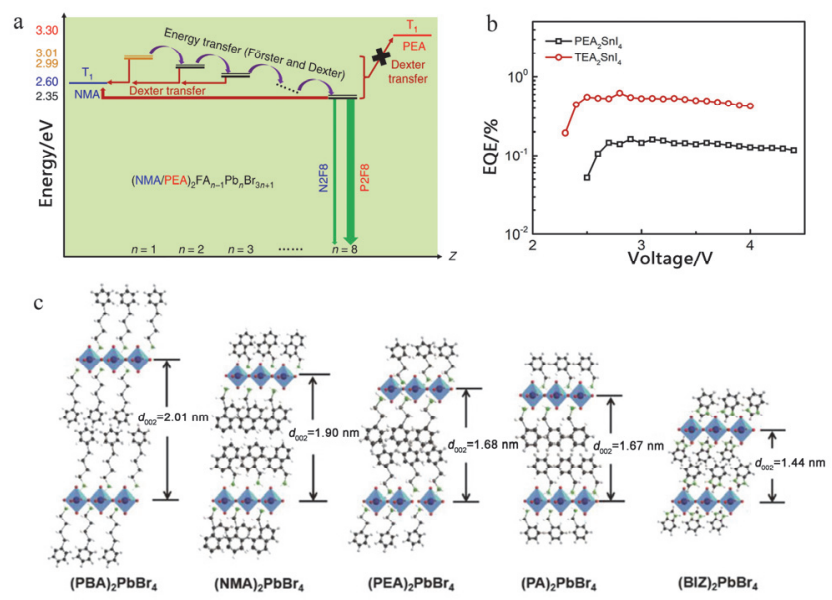

图 6 (a)不同大阳离子体系的准二维钙钣矿中的能量转移过程 ${ }^{[55]}$; (b) 不同大阳离子的锡基钙钛矿 LED 器件性能曲线 ${ }^{[57]}$; (c)不同有机阳离 子对钙钣矿有机插层宽度的影响示意图 ${ }^{[58]}$

Figure 6 (a) Energy transfer mechanism in quasi-two-dimensional perovskite with different spacer molecules ${ }^{[55]}$; (b) EQE characteristics for Sn-based perovskite LED devices with different spacer molecules ${ }^{[57]}$; (c) schematic representation of the barrier width of perovskite films with various organic cations ${ }^{[58]}$ 
钛矿的发光性能有很大影响(图 $6 \mathrm{~b}$ ). 准二维钙钛矿的迁 移率也与大阳离子的选择有关. Wang 等 ${ }^{[58}$ 对比了不同 尺寸的大阳离子在准二维钻钛矿 LED 中的效果, 包括 苯丁铵、菜甲铵、苯乙铵、苯甲铵和苯并咪唑等. 如图 $6 \mathrm{c}$ 所示, 他们发现准二维钙钛矿中有机插层的宽度随 着阳离子尺寸的减小而逐渐减小, 电子和空穴的迁移率 逐渐增大, 更有利于载流子的传输. Ning 等 ${ }^{[59]}$ 对比了以 对苯二甲铵和苯乙铵作为大阳离子的准二维钙钛矿体 系, 通过密度泛函理论计算, 发现在对苯二甲铵形成的 Dion-Jacobson (DJ)型准二维钻钛矿中, 大阳离子与无机 层的结合能以及解离能都比苯乙铵形成的 RuddlesdenPopper (RP) 型钙钛矿大. 这一结果说明二铵形成的 DJ 型钙钛矿晶体结构更稳定, 更能抑制电场下的离子迁 移, 最高效率点的稳定性测试获得了 $100 \mathrm{~h}$ 的半衰期, 体现了较好的稳定性.

此外, 不同类型的大阳离子间的相互作用和堆叠方 式不同, 影响有机相的结晶过程, 决定了准二维钙钛矿 体系中的能量转移过程和发射峰峰位, 因此在制备红、 蓝光 LED 器件时也要考虑大阳离子的选择. 如前所述, 准二维钙钋矿的发射峰取决于体系中最大 $n$ 值的相, 但 是明显的相偏析使得大 $n$ 值的相的带隙接近三维钙钛 矿, 不利于红、蓝光的获得 ${ }^{[43]}$. Sargent 等 ${ }^{[43]}$ 认为苯乙铵 之间较强的范德华力导致有机相 (大阳离子插层)和无机 相 $\left(\left[\mathrm{PbBr}_{6}\right]^{4^{-}}\right)$之间的结晶速率失衡, 造成明显的相偏析. 他们提出异丙铵链长较短, 分子之间范德华力较弱, 用 异丙铵替代部分苯乙铵能改变 $n=1$ 的相的形成能, 减 缓 $n=1$ 的相的形成速率, 获得单分散的相分布, 最终用 纯溴的体系获得发射峰在 $490 \mathrm{~nm}$ 的蓝光准二维钙铁矿 LED 器件. Liao 等 ${ }^{[52]}$ 认为苯乙铵 (PEABr) 与无机八面体 之间较弱的相互作用导致在生长过程中体系 $n$ 值逐渐变 大, 发射峰逐渐红移. 为了获得高亮度的蓝光体系, 他 们提出两点策略: (1)采用对苯二甲铵形成 DJ 型准二维 钙铁矿, 增强大阳离子与无机八面体之间的结合力, 抑 制后续小阳离子进入无机层, 防止 $n$ 值的增大; (2)采用 混合大阳离子的策略, 同时加入对苯二甲铵和苯乙铵, 抑制有机分子之间的堆叠, 减少 $n=1$ 的相的含量, 将 $n$ 值集中在 2 和 3 , 进一步提高了发光效率(图 7a). Liao 等 ${ }^{600]}$ 还提出了另一种双铵离子 $N$-(2-溴乙基)-1,3-丙二胺 (NPA), 如图 7b, 7c 所示, NPA 离子存在两种可能的连接 方式, 因此在混合大阳离子体系中能抑制苯乙胺之间的 堆叠, 又能保持结构的稳定, 获得发射峰为 $485 \mathrm{~nm}$, 外 量子效率为 $2.62 \%$ 的蓝光准二维钻铁矿 LED 器件. 综上 所述, 在制备红、蓝光准二维钻钛矿 LED 器件时, 需要 调节准二维钙钛矿中的相分布并提高相分布的稳定性. 相分布可以通过改变大、小阳离子的比例来调节，而抑 制有机相的快速结晶可通过混合大阳离子的策略来实 现; 提高相分布的稳定性可以通过引入二胺形成 DJ 型 结构来实现.
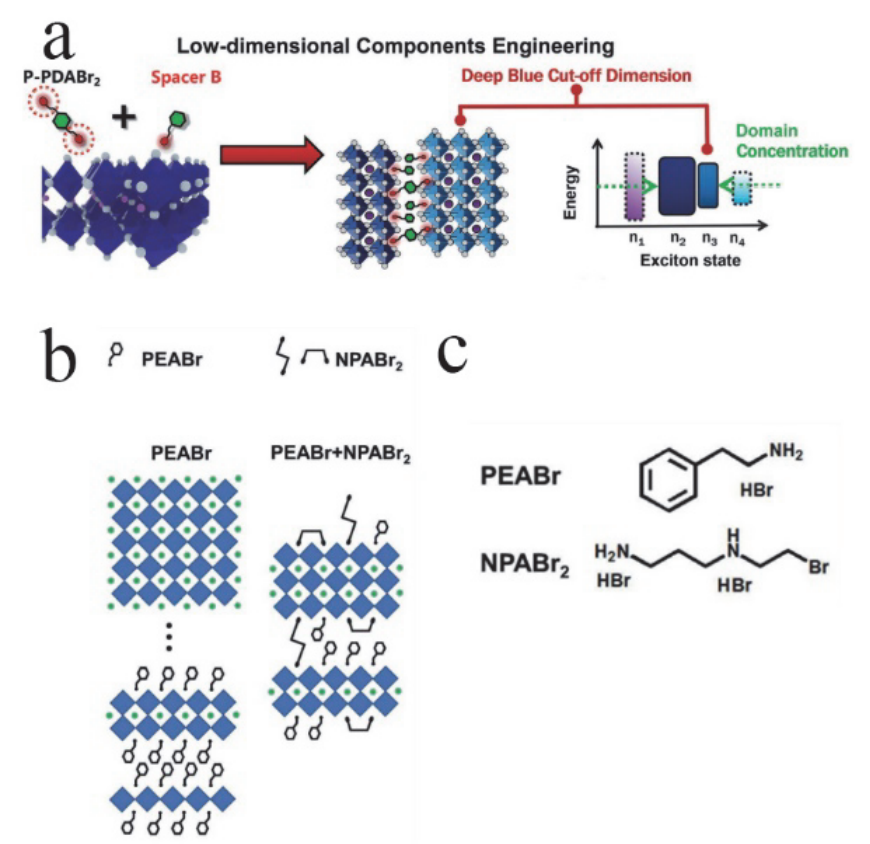

图 7 (a) 低维钻钛矿组分工程示意图 ${ }^{[52]}$; (b) 含有 PEA 或 PEA+NPA 的钙钣矿膜中的异相扩散示意图 ${ }^{[60]}$; (c) PEABr 和 $\mathrm{NPABr}_{2}$ 分子结构 ${ }^{[60]}$ Figure 7 (a) Schematic diagram of low-dimensional perovskite components engineering ${ }^{[52]}$; (b) schematic diagram of different phase diffusion between two perovskite films with PEA or PEA $+\mathrm{NPA}^{[60]}$; (c) molecule structures of $\mathrm{PEABr}$ and $\mathrm{NPABr}_{2}{ }^{[60]}$

综上所述, 在准二维钻钛矿中, 大阳离子不仅是充 当空间插层的作用, 大阳离子本身的性质对准二维钙钛 矿的光电性能、缺陷态、载流子迁移率和结构类型有重 要影响. 含有不饱和键或共轭 $\Pi$ 键的大阳离子自身的三 线态激子能级位置不同, 会影响准二维钙铁矿中激子的 转移过程; 大阳离子形成的空间插层的介电常数不同, 会改变准二维钙钛矿的激子结合能, 从而影响激子辐射 复合的速率; 另外大阳离子的尺寸大小、分子链的刚柔 性会影响准二维钙钋矿的结晶、取向和 $n$ 值分布; 大阳 离子中铵根的数量会改变准二维铝钣矿的结构类型. 因 此, 在制备准二维钙钛矿 LED 器件时, 需要对大阳离子 的种类进行精细调控.

\section{3 钻钛矿量子点}

在钻钛矿量子点器件的制备过程中, 钙钛矿量子点 的合成是关乎最终器件效率的重要环节. 目前合成钙钛 矿量子点的主要两种方法是热注射法 $(\mathrm{HI})^{[6]}$ 和配体辅助 沉淀法(LARP) ${ }^{[62]}$. 热注射法是将阳离子的油酸盐(油酸 铯 Cs-OA 等)溶于高沸点溶剂十八烯, 并注射到溶解了 卤化铅 $\left(\mathrm{PbCl}_{2} 、 \mathrm{PbBr}_{2} 、 \mathrm{PbI}_{2}\right)$ 的十八烯溶液中, 通过控制 温度和反应时间来调节量子点的尺寸 ${ }^{[7,61]}$; 配体辅助沉 淀法是将钙钛矿前驱体和长链有机配体溶解于极性溶 剂二甲基甲酰胺(DMF)中, 并滴入极性较低的溶剂(甲 苯), 通过溶剂溶解度的差异促使钙钣矿量子点的形 成 ${ }^{[62-64]}$. 
钙钛矿量子点合成工艺的优化控制以及量子点组 分的设计极大地影响了钙钛矿 LED 器件的性能. 本小 节主要从组分设计的角度论述钙钛矿量子点 LED 器件 性能优化的方法. 钙钛矿量子点的组分设计主要包括 $\mathrm{ABX}_{3}$ 的组分优化、量子点表面配体的设计以及金属离 子的掺杂这三方面.

对钻钛矿量子点中 $\mathrm{ABX}_{3}$ 的设计主要集中在 $\mathrm{A}$ 位的 阳离子和 $\mathrm{X}$ 位的卤素离子, 主要目的是为了获得特定的 带隙、更好的结晶性、缺陷更少的钙钛矿量子点. $\mathrm{Wu}$

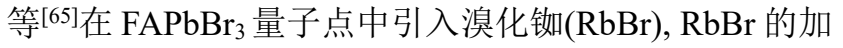
入提高了 $\mathrm{FAPbBr}_{3}$ 量子点的结晶度, 缺陷态密度从 $8.4 \times 10^{17} \mathrm{~cm}^{-3}$ 降低到 $6.1 \times 10^{17} \mathrm{~cm}^{-3}$, 相应薄膜的 PLQY 从 $2 \%$ 提高到 $21.42 \%$, 由此制备的 LED 器件亮度 和电流效率分别提高了 10 倍和 5 倍. Sapra 等[66]将乙铵 阳离子 $\left(\mathrm{EA}^{+}\right)$引入钙钛矿量子点中, 合成了 $\mathrm{EA}_{x} \mathrm{MA}_{1-x^{-}}$ $\mathrm{PbBr}_{3}(x=0 \sim 1)$ 钻钛矿量子点, 通过改变 $\mathrm{EA}^{+}$和 $\mathrm{MA}^{+}$的 比例, 能够在 $2.38 \mathrm{eV}$ 到 $2.94 \mathrm{eV}$ 之间调节量子点的带 隙, 密度泛函理论计算表明体积更大的乙铵离子改变了 $\mathrm{Pb}-\mathrm{Br}$ 键键长, 减小了 $\mathrm{Pb} 6 \mathrm{~s}$ 和 $\mathrm{Br} 4 \mathrm{p}$ 电子的耦合, 从而 增大了带隙(如图 8a). 对 $\mathrm{X}$ 位卤素离子的改变主要是为 了调节钙钛矿量子点的带隙 ${ }^{[61-62]}$. Kido 等 ${ }^{[67]}$ 利用碘化烷 基胺和碘化芳香胺进行阴离子交换, 如图 $8 \mathrm{~b}$ 所示, 从原 始的 $\mathrm{CsPbBr}_{3}$ 量子点获得了 $\mathrm{CsPb}(\mathrm{BrI})_{3}$ 量子点, 发光峰 从 $508 \mathrm{~nm}$ 红移到 $649 \mathrm{~nm}$, 以碘化烷基胺作交换剂的量 子点制备的 LED 器件的 EQE 达到 21.3\%.
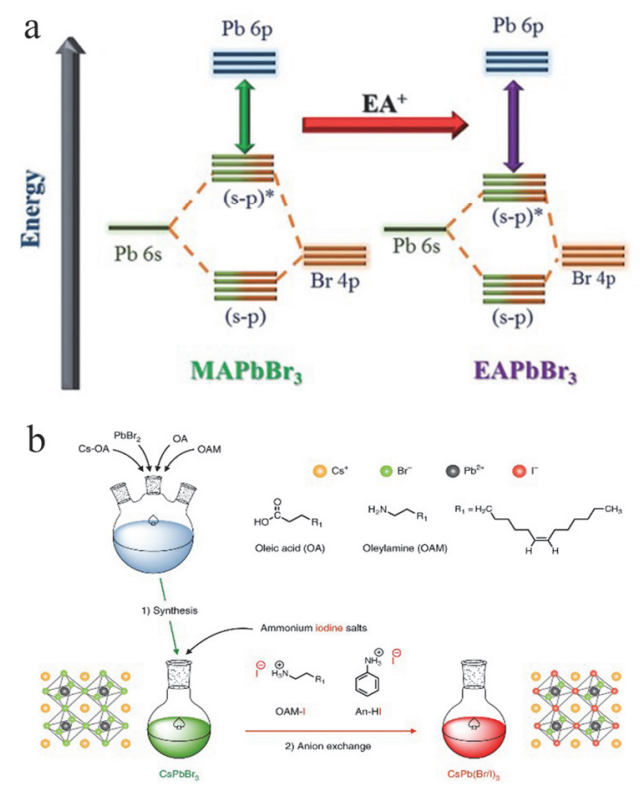

图 8 (a) 在 $\mathrm{MAPbBr}_{3}$ 中引入 $\mathrm{EA}$ 阳离子对 $\mathrm{Pb} 6 \mathrm{p}$ 和 $(\mathrm{Pb} 6 \mathrm{~s}-\mathrm{Br} 4 \mathrm{p})^{\text {* 轨 }}$ 道能量的影响示意图 ${ }^{[66]}$; (b) 用烷基胺和芳香胺对 $\mathrm{CsPbBr}_{3}$ 量子点进 行阴离子交换的示意图(OAM-I, 烷基胺氢磺酸盐; An-HI, 芳香胺氢 碘酸盐) ${ }^{[67]}$

Figure 8 (a) Schematic representation of variation of energy levels of $\mathrm{MAPbBr}_{3}$ in $\mathrm{Pb} 6 \mathrm{p}$ and $(\mathrm{Pb} 6 \mathrm{~s}-\mathrm{Br} 4 \mathrm{p})^{*}$ orbitals on insertion of EA cation ${ }^{[66]}$; (b) scheme of anion-exchange of synthesized pristine $\mathrm{CsPbBr}_{3}$ perovskite QDs using long alkyl ammonium and aryl ammonium. OAM-I, alkyl ammonium; An-HI, aryl ammonium ${ }^{[67]}$
钲钛矿量子点表面的有机配体的选择对量子点的 稳定性、表面的缺陷态密度和成膜后整体的电导率有重 要影响. 在钙钛矿量子点中, 虽然表面长链有机配体很 好地钝化了表面缺陷, 并且量子点分散液能获得接近 $100 \%$ 的苂光量子产率，但是量子点薄膜的苂光产率只 有 $40 \%$ 左右 ${ }^{[68-69]}$. 这是因为在成膜过程中, 有机配体与 量子点表面的结合不稳定, 容易脱落, 形成了很多表面 缺陷 ${ }^{[70-71]}$. 而且长链的有机配体限制了钙钛矿量子点薄 膜的载流子传输性能, 相应的 LED 器件性能较差. Sargent 等 ${ }^{[72]}$ 对量子点表面的有机配体进行优化, 用一种溴 盐(十二烷基二甲基溴化铵, DDAB)替代表面部分长链 油胺(图 9a). DDBA 钝化了表面缺陷, 减少了溴空位的 产生，配体交换后的量子点的 PLQY 从 49\%提高到 $71 \%$, 在量子点薄膜的导电性测试中, 交换后的样品电流提高 了一个数量级, 显示出更好的载流子传输, LED 器件效 率也从 $0.1 \%$ 提高到了 $3 \%$. Zeng 等 ${ }^{[73-75]}$ 对钙钛矿量子点 表面的配体进行了一系列的优化. 根据极性大小对不同 溶剂进行笁选，选择己烷/乙酸乙酯的混合溶剂对量子 点表面的有机配体进行 “清洗”，适当降低表面配体的 密度，以获得量子点表面钝化和载流子注入两方面的平 衡, 获得量子点分散液稳定、PLQY 高、载流子注入好 的钻钛矿量子点薄膜(图 9b), 相应 LED 器件的 EQE 提

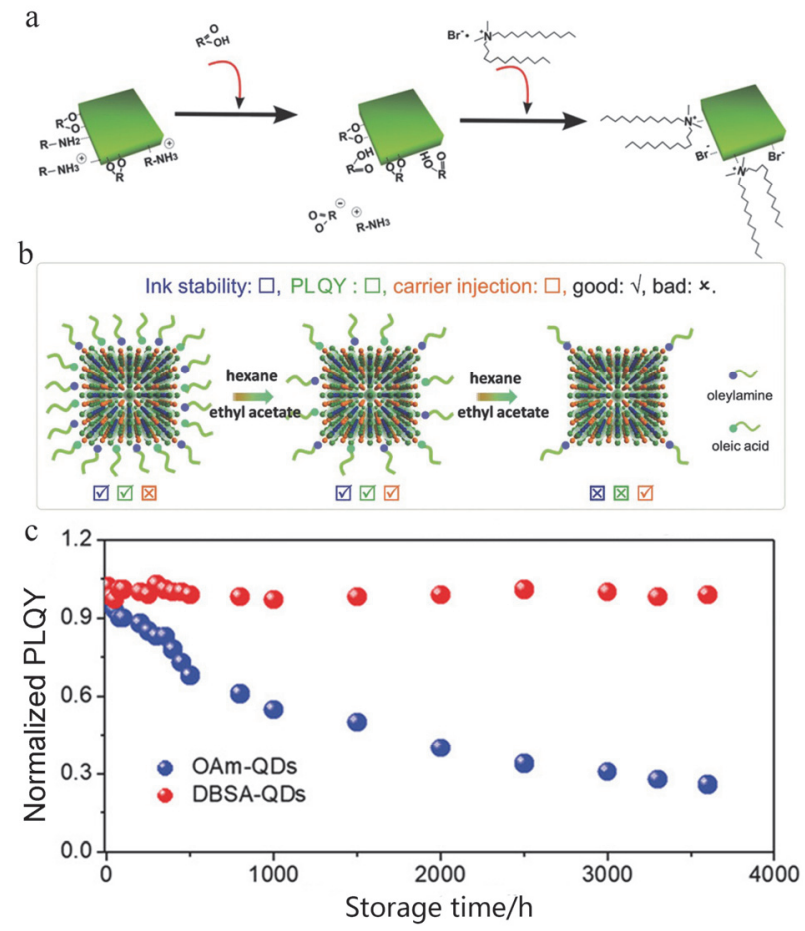

图 9 (a) $\mathrm{CsPbBr}_{3}$ 量子点表面的配体交换机理 ${ }^{722]}$; (b) $\mathrm{CsPbBr}_{3}$ 量子点 表面配体密度和相应的分散液稳定性、PLQY 和载流子注入能力 ${ }^{[73]}$; (c) 不同配体策略的量子点的 PLQY 随储存时间的变化 ${ }^{[75]}$

Figure 9 (a) The ligand-exchange mechanism on $\mathrm{CsPbBr}_{3}$ QD surfaces $^{[72]}$; (b) schematic illustration of the control of ligand density on $\mathrm{CsPbBr}_{3}$ QD surfaces and the corresponding changes of ink stability, PLQY and carrier injection ${ }^{[73]}$; (c) PLQY variation of the above corresponding samples after different storage times ${ }^{[75]}$ 
高了 50 倍, 达 $6.27 \%$ [73]. 随后 Zeng 等 ${ }^{[74]}$ 对 $\mathrm{CsPbBr}_{3}$ 量 子点的表面配体进行有机-无机杂化, 在有机配体(辛酸 根、DDAB)中掺入无机配体(金属溴盐), 基于此的钙钛 矿量子点 LED 器件的外量子效率达到 $16.48 \%$. 其中有 机配体保持了量子点分散液的稳定性, 而无机配体有两 个作用: (1)提高纳米晶表面溴离子含量, $n(\mathrm{Br}): n(\mathrm{Cs})$ 的 比例从 2.94 提高到 3.45 , 减少了卤素空位缺陷的数量; (2)取代部分有机配体提高了体系的导电性, 更有利于 载流子的注入与传输. 此后, Zeng 等[75]又提出 “溴等价” 配体(Br-equivalent ligand)的策略, 采用长链苯磺酸作为 配体, 由于磺酸根与铅之间较强的作用力, 长链苯磺酸 能够在量子点表面形成稳定的配体, 并且能消除表面的 溴空位. 此种方法制备的量子点的稳定性大大提高, 经 过多次提纯、高强度光照以及五个月的储存后仍能保持 $90 \%$ 的 PLQY(图 9c). Sun 等 ${ }^{[76]}$ 提出一种 “缺有机配体” (organic-ligand-lacking)的纳米晶制备方法, 利用亚砜卤 化物与纳米晶表面的油酸、油胺配体间的反应, 去除纳 米晶表面大量的有机配体, 并且提供过量的卤素离子来 钝化纳米晶表面的卤素空位缺陷. 此种方法制备的 $\mathrm{CsPbCl}_{3}$ 和 $\mathrm{CsPbBr}_{3}$ 纳米晶的 PLQY 达 $80 \%$ 以上, 并且 器件电流-电压测试表明有机配体的减少提高了纳米晶 薄膜的电导率, 最终获得了发射波长为 $460 \mathrm{~nm}$, 外量子 效率为 $1.35 \%$ 的蓝光 LED 器件.

有机配体的调控是对量子点表面缺陷的钝化以及 整体电导率的改进, 为了进一步提高钻钛矿量子点 LED 器件的性能, 对材料本征性质的优化和设计也是 可行的方法. 在传统的 II -VI族和III- V 族量子点中, 杂 质掺杂是调节半导体材料光电性质的重要手段 ${ }^{[77-79]}$. 杂 质掺杂可以影响材料的 pn 性、磁性以及苂光发射, 因此 金属离子的掺杂逐渐发展为调节钻钛矿量子点光电性 能的重要手段. $\mathrm{Mn}^{2+}$ 是在 $\mathrm{CsPbX}_{3}(\mathrm{X}=\mathrm{Cl}, \mathrm{Br}, \mathrm{I})$ 体系中 常用的掺杂金属 ${ }^{[80-83]}$. Chen 等 ${ }^{[80]}$ 用 $\mathrm{Mn}^{2+}$ 替代 $\mathrm{CsPbX}_{3}$ 中 的部分 $\mathrm{Pb}^{2+}$, 以稳定 $\mathrm{CsPbX}_{3}$ 的晶格, 通过 HAADFSTEM 和 EDS 元素分布证实, $\mathrm{Mn}^{2+}$ 成功掺入 $\mathrm{CsPbX}_{3}$ 晶 格中, 并且在量子点中均匀分布. 第一性原理计算结果 显示, $\mathrm{CsPbBr}_{3}: \mathrm{Mn}$ 的形成能比无掺杂的高 $0.32 \mathrm{eV}$, 表 明在 $\mathrm{CsPbX}_{3}$ 中, $\mathrm{Mn}^{2+}$ 替代部分 $\mathrm{Pb}^{2+}$ 在热力学上是更稳 定的. 相应的量子点薄膜在空气中的稳定性测试表明 $\mathrm{Mn}^{2+}$ 掺杂提高了 $\mathrm{CsPbBr}_{3}$ 量子点的稳定性: 添加了 $\mathrm{Mn}^{2+}$ 的膜存放 $120 \mathrm{~d}$ 后仍保持 $60 \%$ 的初始荧光强度, 而 未掺杂的样品在 $30 \mathrm{~d}$ 后就失去苂光. 以 $\mathrm{Mn}^{2+}$ 掺杂的量 子点为发光层制备的 LED 器件效率达到 $1.49 \% . \mathrm{Yu}$ 等 ${ }^{[84]}$ 通过热注入的方法在 $\mathrm{CsPbBr}_{3}$ 量子点中掺入 $\mathrm{Ce}^{3+}$ 离子, $\mathrm{Ce}^{3+}$ 的掺入使量子点的 PLQY 从 $41 \%$ 提高到 $89 \%$. 瞬态 吸收光谱表明掺杂之后的量子点中激子的弛豫加快, 而 且瞬态苂光表明苂光寿命降低. 作者认为 $\mathrm{Ce}^{3+}$ 掺杂增 加了 $\mathrm{CsPbBr}_{3}$ 导带附近的态密度, 提供了更多辐射复合 的通道, 从而影响了苂光动力学, 辐射复合的速率加快. 掺杂之后的 LED 器件的 EQE 从 $1.6 \%$ 增加到 $4.4 \%$.
Rogach 等 ${ }^{[85}$ 在 $\mathrm{Ag} / \mathrm{ZnO}$ 基底上制备 $\mathrm{CsPbI}_{3}$ 量子点薄膜, 他们发现 $\mathrm{Ag}^{+}$会从基底向上扩散并逐渐掺入 $\mathrm{CsPbI}_{3}$ 晶 格中. 扩散的 $\mathrm{Ag}^{+}$能钝化 $\mathrm{CsPbI}_{3}$ 的表面缺陷, 并且针定 于表界面的 $\mathrm{Ag}$ 或 $\mathrm{AgI}$ 能提高量子点的稳定性. $\mathrm{Ag}^{+}$的掺 入使量子点薄膜的 PLQY 从 $60 \%$ 提高到 $81 \%$, 相应的 LED 器件效率从 $7.3 \%$ 提高到 $11.2 \%$. Rogach 等 ${ }^{[86]}$ 在 $\mathrm{CsPbI}_{3}$ 量子点中引入 $\mathrm{SrCl}_{2}, \mathrm{Sr}^{2+}$ 的离子半径(118 pm) 比 $\mathrm{Pb}^{2+}$ (119 pm)略小, 所以 $\mathrm{Sr}^{2+}$ 的掺入会造成轻微的晶格 收缩, 增大了钙钛矿的形成能, 提高了 $\mathrm{CsPbI}_{3}$ 的稳定性 (在空气中保存 $60 \mathrm{~d}$ 仍保持黑相); 此外, $\mathrm{Cl}^{-}$能钝化晶体 表面的卤素空位缺陷, 减少非辐射复合位点, PLQY 从 $65 \%$ 提高到 $84 \%$, LED 器件效率提高了两倍, 达到 $13.5 \%$.

本小节主要从 $\mathrm{ABX}_{3}$ 的组分优化、量子点表面配体 的设计以及金属离子的掺杂这三方面论述了组分设计 对钙钛矿量子点 LED 器件性能的影响. $\mathrm{ABX}_{3}$ 的组分优 化和金属离子的掺杂主要涉及钙钛矿材料的本征性质 的调节, 表面配体的优化则更加能体现量子点这种低维 材料的特点和优势. 因此, 我们认为对表面配体的优化 和调节将是获得尺寸和形状单分散、表面性质稳定的钙 钛矿量子点 LED 器件的关键.

\section{4 缺陷钝化对钙钛矿 LED 器件性能的影响}

有机-无机杂化钙钛矿是一种离子晶体, 相应的离 子键键能较低, 容易产生各种点缺陷, 虽然理论计算表 明钙钛矿中形成的缺陷大部分是浅能级缺陷, 但是大量 的实验证明钝化钙钛矿中的缺陷是提高光电器件性能 的重要途径之一 [87-91]. 缺陷的钝化对提升 LED 器件的 稳定性也很重要, 因为 LED 器件在工作条件下的外加 电场强度较大, 会加速缺陷的迁移和扩散, 进一步破坏 钙钛矿, 加速器件性能的衰减. 本章节根据钝化分子的 类型，从路易斯碱、碱金属盐和有机盐等角度详细论述 缺陷钝化对钙钛矿 LED 器件性能和稳定性的影响.

\section{1 路易斯碱}

铅卤钙钛矿是离子晶体, 晶体中的离子键键能较 低，因此在晶界和薄膜表面处会存在大量未配位的离 子 ${ }^{[88]}$. 其中, 卤素空位的出现会导致钙钛矿中未配位的 铅形成铅悬挂键, 成为非辐射复合中心, 需要负电性的 基团来消除. 路易斯碱中存在未成键的孤对电子，可以 与未配位的铅配位, 针化卤素空位形成的缺陷, 是铅卤 钙钛矿中常用的针化材料 ${ }^{[90,92-94]}$. Huang 等 ${ }^{[14,95]}$ 在钙钛 矿体相和下界面处引入 5-氨基戊酸(5AVA), 如图 10a 所 示, 5AVA 中的羧基与底部传输层的修饰分子结合, 胺基 裸露在表面. 胺基一方面提高基底的浸润性，改善钙钛 矿的成膜性; 另一方面能够针化钙钛矿下表面的未配位 铅缺陷. 钙钛矿薄膜获得 70\%的 PLQY, 相应的近红外 光的 LED 器件的 EQE 达到 $20.7 \%$. Gao 等 ${ }^{[5]}$ 发现在 $\mathrm{FAPbI}_{3}$ 体系中, 钝化剂长链胺分子中的氮原子的给电子 
能力较强, 虽然作为路易斯碱能钝化未配位的铅悬挂 键, 但是胺分子中的氮原子也会和 $\mathrm{FA}^{+}$离子中的氢形成 氢键, 导致胺分子无法完全发挥钝化的作用. 因此, 他 们设计了一系列胺分子(图 10b), 通过在有机链中引入 氧原子, 利用氧原子的吸电子诱导效应降低氮原子上的 电子密度, 并调节链长来改变诱导效应的强弱, 获得了 一个不易形成氢键, 更容易参与缺陷钝化的胺分子 (ODEA). 用这种分子进行钝化的钙钛矿薄膜制备的近 红外钲钛矿 LED 器件的 EQE 达到 $21.6 \%$. You 等 ${ }^{[25]}$ 在准 二维钙钛矿与电子传输层之间加入一层三正辛基氧膦 (TOPO) 的插层, 有效地钝化了钙钛矿表面的缺陷. TOPO 针化之后的钙钛矿薄膜的 PLQY 从 57.3\%提高到 $73.8 \%$, 绿光 LED 器件的外量子效率从 $12.12 \%$ 提高到 $14.36 \%$. 在 $\mathrm{CsPbBr}_{3}$ 体系中, 研究者们在前驱体中加入 聚环氧乙烷(PEO) ${ }^{[39,96]}$. PEO 一方面在结晶过程中增加 成核位点, 减小晶粒尺寸; 另一方面氧原子对钙钛矿表 界面的缺陷也有钝化作用. 添加了 $\mathrm{PEO}$ 的 $\mathrm{CsPbBr}_{3}$ 薄膜 的 PLQY 从 $7 \%$ 提高到 $60 \%{ }^{[96]}$.

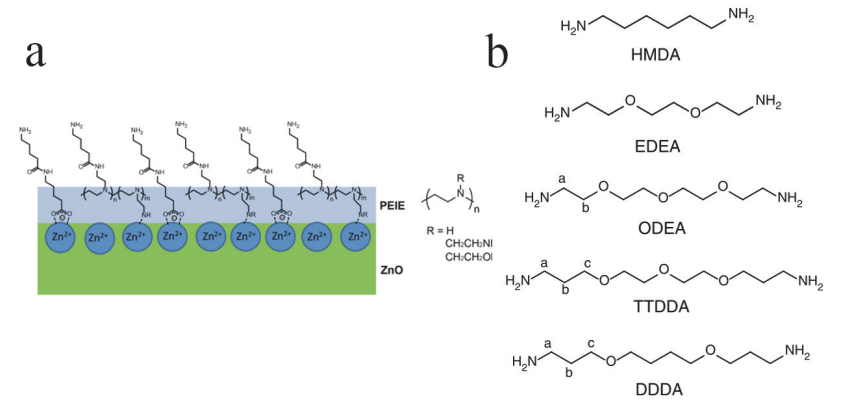

图 10 (a) $5 \mathrm{AVA}$ 在氧化锌表面的反应以及构型示意图 ${ }^{[14]}$; (b) 针化分 子结构 $[5]$

Figure 10 (a) Dehydration reaction of 5AVA on top of the ZnO-PEIE surface and configuration of 5AVA on the $\mathrm{ZnO}$ surface ${ }^{[14]}$; (b) the molecular structure of passivating moleculars ${ }^{[5]}$

缺陷的存在一方面不利于辐射复合, 降低器件效 率, 另一方面在电场作用下, 缺陷会加快离子迁移和钙 钛矿本身的破坏, 因此路易斯碱对缺陷的钝化除了能提 高器件效率, 还能有效提高器件的稳定性. Song 等 ${ }^{[97]}$ 在 $\mathrm{MAPbBr}_{3}$ 钙钛矿表面引入了乙二胺分子(EDA)和支状聚 乙烯二胺(PEI), 发现这两个带胺基的分子都能抑制钙 钛矿非辐射复合, 并且抑制电极被钙钛矿中迁移的离子 腐蚀. 在恒定电流密度 $20 \mathrm{~mA} \cdot \mathrm{cm}^{-2}$ 下, 没有胺分子钝 化的器件在 $2500 \mathrm{~s}$ 内衰减到初始亮度的 $10 \%$, 而针化之 后的器件在 $14000 \mathrm{~s}$ 之后仍保持初始亮度的 $70 \%$ (如图 11a). Wang 等 ${ }^{[41]}$ 在 $\mathrm{FAPbI}_{3}$ 钙钛矿表面旋涂了一层茮胺 分子(BA), BA 能钝化表面缺陷, 抑制离子迁移 ${ }^{[98-99]}$, LED 器件在 $100 \mathrm{~mA} \cdot \mathrm{cm}^{-2}$ 的高电流下的半衰期从 $0.5 \mathrm{~h}$ 提高到 $23.7 \mathrm{~h}$. Sargent 等 ${ }^{[100]}$ 认为钙钛矿晶界处的铅悬挂 键会吸附氧分子, 在钙钛矿受到光激发或电激发后, 电 子容易转移到氧分子形成超氧根 $\left(\mathrm{O}_{2}{ }^{-}\right)$, 进一步促使钙 钛矿的氧化和分解. 为了提高 LED 器件的稳定性, 他们
采用三苯氧膦(TPPO)对准二维钙钛矿表面进行钝化, 以减少铅悬挂键以及由此引起的钙钛矿的分解. 在空气 中连续光照下的稳定性测试表明，经 TPPO 处理的钙钛 矿经历了 $300 \mathrm{~h}$ 光照后，荧光亮度和发射峰位仍保持不 变, 而未处理的钙钛矿在 $1 \mathrm{~h}$ 后衰减到 $40 \%$, 并且伴有 发射峰的变宽和红移. 相应的 LED 器件在初始亮度为 $4000 \mathrm{~cd} \cdot \mathrm{m}^{-2}$ 的半衰期从 $53 \mathrm{~s}$ 提高到 $44 \mathrm{~min}$, 在减小钝化 分子的空间位阻后, 进一步将半衰期提高到 $3.5 \mathrm{~h}$ (图 11b).
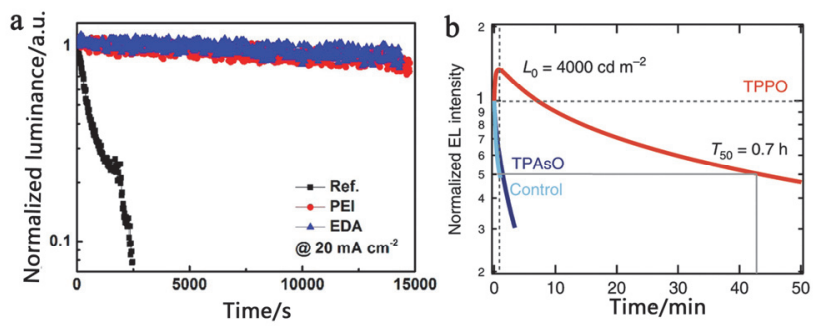

图 11 (a) 针化前后封装的钲钛矿 LEDs 在环境条件下归一化的亮度 随器件工作时间的变化 ${ }^{[97]}$; (b)初始亮度为 $4000 \mathrm{~cd} \cdot \mathrm{m}^{-2}$ 的条件下, 未 处理的和边缘稳定的钻钣矿 LED 器件操作稳定性曲线 ${ }^{[100]}$

Figure 11 (a) Normalized luminances of encapsulated PeLEDs with and without passivation under ambient conditions as functions of operation time ${ }^{[9]}$; (b) operational device stability of untreated controls and edge-stabilized perovskite LEDs at a starting luminance of $4000 \mathrm{~cd} \cdot$ $\mathrm{m}^{-2[100]}$

\section{2 碱金属盐}

碱金属盐在针化钙钛矿缺陷方面有很多报道，特别 是碘化钾的加入能有效地抑制表界面卤素空位缺陷的 形成，以及抑制卤素离子的迁移，这对钻钛矿 LED 器件 性能和稳定性的提升有很大启发 ${ }^{[89,101]}$. Prezhdo 组 ${ }^{[102]}$ 通 过理论计算发现，在钙钛矿中掺入碱金属离子能显著增 加碘填隙缺陷的形成能，降低缺陷浓度; 而且碱金属离 子与体系中已存在的碘填隙缺陷紧密结合, 消除由此产 生的缺陷态，将载流子寿命提高 7 倍. Stranks 等 ${ }^{[89]}$ 在 $\left(\mathrm{Cs}_{0.06} \mathrm{FA}_{0.79} \mathrm{MA}_{0.15}\right) \mathrm{Pb}\left(\mathrm{I}_{0.85} \mathrm{Br}_{0.15}\right)_{3}$ 的混合卤素体系中加入 碘化钾 $(\mathrm{KI})$, 如图 $12 \mathrm{a}$ 所示: 过量的碘离子能消除卤素 空位缺陷; 钾离子在钙钛矿多晶膜的表界面与过量的卤 素形成较稳定的钾卤素盐，抑制卤素的迁移. Sun 等[103] 在 $\mathrm{CsPbBr}_{3}$ 体系中加入溴化锂 $(\mathrm{LiBr})$, 获得了 $\mathrm{EQE}$ 达 $16.2 \%$ 的绿光 LED 器件. 他们认为, 如图 $12 \mathrm{~b}$ 所示: 卤素 过量的体系能有效抑制卤素空位缺陷的形成，而体积较 小的锂离子存在于钙钛矿晶体的表界面, 不会进入晶格 影响钙钛矿的能级结构和结晶性. Yao 等 ${ }^{[104]}$ 在制备 $\mathrm{CsPbI}_{3-x} \mathrm{Br}_{x}$ 纳米晶时加入油酸钾, 获得了溴化钾表面钝 化的纯红光钙钛矿纳米晶 LED 器件, 效率达 $3.55 \%$. 钾 离子在钙钛矿纳米晶表面与溴离子结合，抑制了表面卤 素空位缺陷的形成, 以及卤素离子的迁移和相分离, 提 高了纯红光(637 nm)发射峰的稳定性(图 12c).

碱金属卤素盐的加入不仅能提供富卤素的化学环 境，抑制钙钛矿中卤素空位的产生，而且碱金属离子与 卤素离子结合能有效地抑制电场作用下卤素离子的迁 
移. 因此, 加入碱金属卤素盐也能有效地提高钙钛矿 LED 器件的稳定性. Zhao 等 ${ }^{[105]}$ 在 $\mathrm{FAPbI}_{3}$ 体系中同时加 入碘化铯和碘化铷两种碱金属碘盐, 通过 DFT 计算和 深度分辨 $\mathrm{X}$ 射线光电子能谱(XPS)分析发现, 铯离子均 匀地分布在钙钛矿体相中, 而铷离子主要分布在钙钛矿 表面. 计算结果进一步表明, 铯、铷的加入使周围碘离 子的带电量平均增加了 $4.6 \%$ 和 $5.9 \%$, 因此碘离子与 $\left[\mathrm{PbI}_{6}\right]^{4^{-}}$无机框架之间的库仑作用力增强, 抑制了电场 作用下碘离子空位的形成, 提高了器件的稳定性.
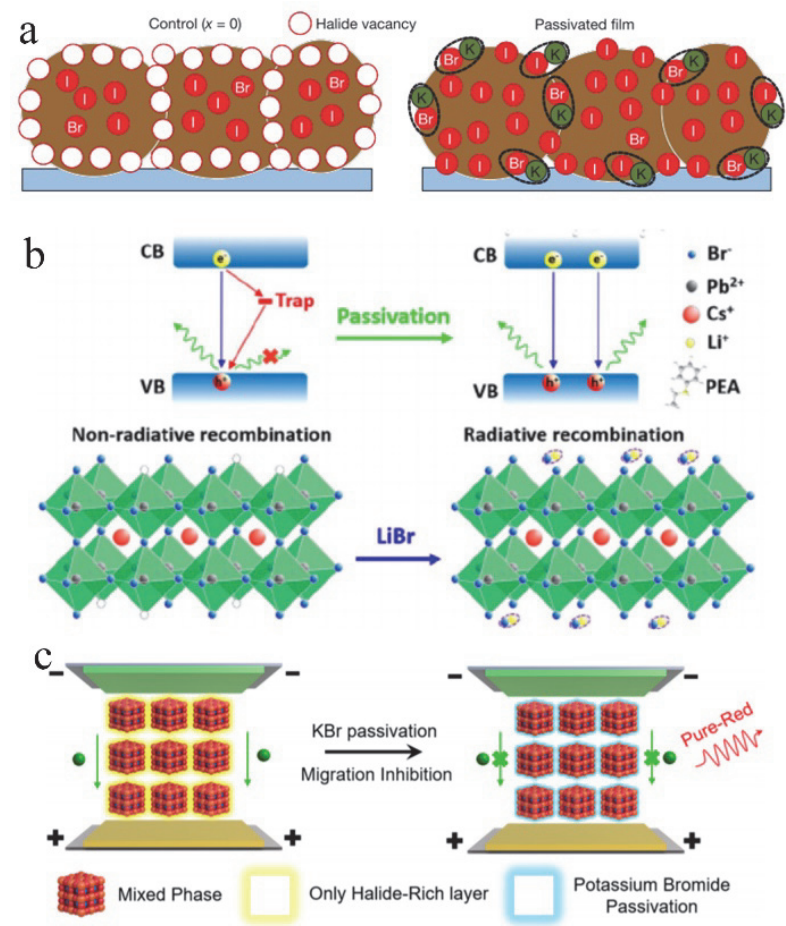

图 12 (a) 过量卤素离子对卤素空位缺陷的调控的截面示意图, $\mathrm{K}^{+}$离 子在晶界和表面与卤素形成良性的化合物, 固定了过剩的卤素 ${ }^{[89]}$; (b) $\mathrm{CsPbBr}_{3}$ 钙铁矿薄膜表面卤素空位缺陷和无机分子 $\mathrm{LiBr}$ 针化示意 图 ${ }^{[103]}$; (c) 富 $\mathrm{KBr}$ 的表面针化抑制离子迁移示意图 ${ }^{[104]}$

Figure 12 (a) Schematic of a cross-section of a film showing halide-vacancy management in cases of excess halide, in which the surplus halide is immobilized through complexing with potassium into benign compounds at the grain boundaries and surfaces ${ }^{[89]}$; (b) a schematic depicting the surface halide vacancies of $\mathrm{CsPbBr}_{3}$ perovskite films and passivated by inorganic molecules of $\mathrm{LiBr}^{[103]}$; (c) illustration of ion migration suppressed by $\mathrm{KBr}$-enriched surface passivation ${ }^{[104]}$

\section{3 有机盐}

钙钛矿中带电的缺陷态不仅可以通过路易斯酸碱 来钝化, 有机盐中带电的官能团也可以消除阳离子空 位、卤素空位形成的缺陷态，起到钝化作用. 例如 Huang 等 ${ }^{[106]}$ 设计了一种钝化分子 D4TBP, 如图 13a 所示, 其中 带负电的羧基可以钝化正电性的卤素空位缺陷, 带正电 的胺基钝化负电性的阳离子空位缺陷, 相应的钻钛矿太 阳能电池获得了 $1.23 \mathrm{~V}$ 的开路电压, 该策略也可以应用 到钙钛矿 LED 器件中. Zhao 等[107]通过有限元分析模拟 了钻钛矿 LED 器件工作中的载流子复合过程, 研究发
现钻钛矿上界面是非辐射复合发生的主要场所，因此在 钙钛矿上表面旋涂了一层碘化苯乙胺盐(PEAI); PEAI 中 的碘离子能针化表面的碘离子空位缺陷, 而 $\mathrm{PEA}^{+}$阳离 子钝化表面的阳离子空位缺陷, 钝化后的器件不仅效率 有很大提升, 且 $100 \mathrm{~mA} \cdot \mathrm{cm}^{-2}$ 恒电流下的半衰期从 $1.5 \mathrm{~h}$ 提高到 $11.3 \mathrm{~h}$ (图 13b). Xiao 等 ${ }^{[108]}$ 设计了一种有机盐 (FPMATFA), 阳离子为 4-氟苯甲胺(FPMA), 阴离子为 三氟乙酸根(TFA). 其中 4-氟苯甲胺能和表界面的卤素 悬挂键结合, 针化卤素缺陷; 三氟乙酸根能和未配位的 铅结合, 消除铅悬挂键(图 13c). 钝化后的钙钛矿 LED 器件效率达到 $20.9 \%, 2.5 \mathrm{~mA} \cdot \mathrm{cm}^{-2}$ 恒定电流下的半衰期 从 $0.25 \mathrm{~h}$ 提高到 $14 \mathrm{~h}$.
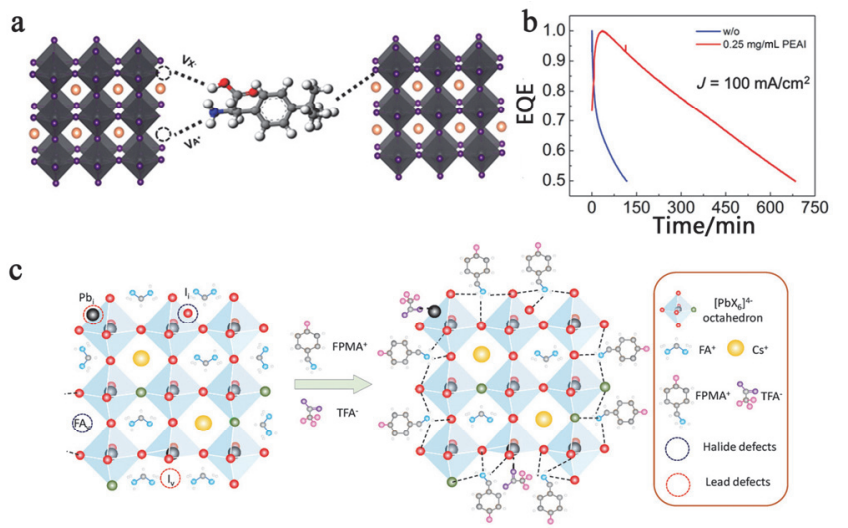

图 13 (a) D4TBP 分子对不同缺陷位点的针化机理示意图 ${ }^{[106]}$; (b) 有 无 PEAI 层的钙铁矿 LED 器件的工作稳定性 ${ }^{[107]}$; (c) FPMATFA 有机盐 对钙铁矿晶界的缺陷钝化示意图 ${ }^{[108]}$

Figure 13 (a) Schematic illustration of the origin of D4TBP passivation effect on different defect sites $^{[106]}$; (b) operated stability of the PeLED devices with and without PEAI layer ${ }^{[107]}$; (c) schematic illustration of defect passivation at perovskite grain boundaries by FPMATFA ${ }^{[108]}$

本节中，我们根据钝化分子的不同，讨论了不同类 型的钝化分子发挥钝化作用的机理和效果, 缺陷的钝化 很大程度上抑制了非辐射复合的发生，提高了钙钛矿薄 膜的苂光效率以及 LED 器件的外量子效率. 此外, 钝化 后的 LED 器件的稳定性都有很大提升, 这是因为在器 件工作时，外电场作用下缺陷附近的离子迁移会加剧; 而且 LED 器件在工作时会产生热量 ${ }^{[109]}$, 器件温度的上 升会进一步激发缺陷诱导的离子迁移, 导致缺陷的扩散 和钙钛矿的破坏. 因此, 缺陷钝化对 LED 器件稳定性的 提高至关重要. 此外钝化分子的设计除了需要满足对不 同类型的缺陷的钝化，还需要考虑对钲钛矿薄膜电导率 的影响. 大量有机组分的加入虽然能起到很好的针化和 限域作用，但是有机分子会降低钙钛矿的载流子迁移 率, 导致器件工作时产生更多的热能, 不利于器件的稳 定性. 良好的钝化分子需要具备较高的载流子迁移率, 以保持 LED 器件较好的载流子注入和传输; 同时需要 具有较宽的带隙, 从而与钙钛矿形成量子阱, 防止注入 钲钛矿中的载流子被钝化分子转移. 


\section{5 界面修饰对钻钛矿 LED 器件性能的影响}

发光二极管是由多种功能层依次沉积形成的多层 器件, 不同功能层之间的界面性质会影响器件工作过程 中的各物理过程. 界面修饰的作用不能一概而论, 其作 用包括提高前驱液在基底上的浸润性、提高钙钛矿的成 膜性、钝化上下界面的缺陷、阻挡载流子的注入或泄露、 降低载流子的注入势垒等.

Huang 等 ${ }^{[13]}$ 在 $\mathrm{ZnO}$ 电子传输层和钻铁矿之间引入 一层聚乙烯亚胺(PEI), PEI 提供了一个亲水性的表面, 有利于形成高质量的钻钛矿薄膜; 且 PEI 将 $\mathrm{ZnO}$ 的表面 功函从 $3.7 \mathrm{eV}$ 降低到 $3.2 \mathrm{eV}$, 有效提高电子的注入. Rogach 组 ${ }^{[10]}$ 发现在 ITO/PEDOT:PSS/Poly-TPD/CsPbBr 3 纳米晶 $/ \mathrm{TPBi} / \mathrm{LiF} / \mathrm{Al}$ 的器件结构中, 空穴传输层 Poly-TPD 和钙钛矿之间的注入势垒达 $1.09 \mathrm{eV}$, 限制了 空穴的注入, 因此, 他们在空穴传输层和钙钛矿之间引 入一层分子尺度薄的全氟化离子聚合物(PFI), 由于 PFI 在表面形成偶极, 使空穴传输层的价带下移 $0.34 \mathrm{eV}$, 有 效地降低空穴的注入势垒; 随后 Rogach 等 ${ }^{[111]}$ 又发现 $\mathrm{CsPbBr}_{3}$ 的价带和电子传输层 TPBi 的 HOMO 能级的能 量差只有 $0.02 \mathrm{eV}$, 导致 TPBi 阻挡空穴能力较弱, 容易 形成漏电流. 因此, 在 $\mathrm{CsPbBr}_{3}$ 纳米晶薄膜上表面引入 一层笼型聚倍半硅氧烷(POSS), 能够有效地阻挡空穴的 泄露, 将载流子集中在钻钛矿发光层, 有助于其辐射复 合. $\mathrm{You}$ 等 ${ }^{[12]}$ 在 $\mathrm{ZnO}$ 和 $\mathrm{CsPbBr}_{3}$ 之间引入一层聚乙烯吡 咯烷聚合物(PVP), PVP 提供一个亲水的表面, 有助于获 得无孔洞、高质量的钻铁矿薄膜; 并且 PVP 能同时针化 $\mathrm{ZnO}$ 表面的缺陷和钙钛矿下界面的缺陷, 绝缘性的 PVP 能适当抑制电子的注入, 改善载流子的注入平衡. Wei 等 ${ }^{[6]}$ 在 $\mathrm{CsPbBr}_{3}$ 和电子传输层界面引入一层聚甲基丙烯 酸甲酯(PMMA), 绝缘性的 PMMA 能阻挡电子的注入, 使得电子和空穴之间的注入更加平衡, 绿光 LED 器件 获得了 $20.3 \%$ 的外量子效率. Choy 等 ${ }^{[113]}$ 在空穴传输层 $\mathrm{NiO}_{x}$ 表面引入一层聚苯乙烯磺酸钠(PSSNa), PSSNa 能 钝化 $\mathrm{NiO}_{x}$ 表面的缺陷, 提高浸润性, 改善钙钛矿薄膜的 形貌, 且 $\mathrm{PSSNa}$ 在 $\mathrm{NiO}_{x}$ 表面形成偶极, 使空穴注入势垒 降低了 $0.34 \mathrm{eV}$, 有效地促进了空穴的注入. Meng 等[114] 在电子传输层 TPBi 和金属阴极银之间插入一层稀土金 属镱 $(\mathrm{Yb}), \mathrm{Yb}$ 能显著降低金属电极的功函, 获得的 $\mathrm{Yb} / \mathrm{Ag}$ 电极的功函为 $2.6 \mathrm{eV}$, 与 TPBi 的 LUMO 能级 2.7 $\mathrm{eV}$ 匹配, 降低了电子注入势垒, 平衡了载流子的注入. 不平衡的载流子注入会导致界面处载流子富集, 在高电 流下引起俄歇复合, 导致效率滚降的发生. $\mathrm{Yb}$ 插层的加 入显著缓解了效率在高电流、高亮度下的滚降, 2000 $\mathrm{cd} \cdot \mathrm{m}^{-2}$ 时器件的电流效率为 $22 \mathrm{~cd} \cdot \mathrm{A}^{-1}$, 在 $5000 \mathrm{~cd} \cdot \mathrm{m}^{-2}$ 的效率仍有 $16.8 \mathrm{~cd} \cdot \mathrm{A}^{-1}$.

钻钛矿 LED 器件的稳定性是其实现商业化之前必 须要解决的问题, 虽然目前的结果距离商业化的水平还 有很长的路要走, 但是对钻钋矿缺陷的针化和器件功能
层界面的修饰能大幅提高器件的稳定性. 对钙钛矿 LED 器件稳定性的研究可以从器件和材料两方面来考 虑: 器件方面主要是指制备工艺、载流子平衡、外部水 氧的隔绝、器件工作时的温度变化等; 材料方面主要是 指材料杂质、非辐射复合中心、光和电作用引发的材料 退化等. 在这些因素中, 钻钛矿 LED 器件最难解决的问 题主要是非辐射复合中心的累积和由此引发的更严重 的离子迁移. 这是因为有机无机杂化钙钛矿是离子化合 物, 组成钙铁矿的离子带电量低, 离子半径大, 离子键 键能较低, 在电场作用下容易发生断键, 最终导致离子 在电场下的漂移, 形成空位、间隙等缺陷，造成非辐射 复合中心的累积. 我们认为缺陷的存在提供了离子迁移 的通道, 更容易促使离子的移动, 因此对钙钛矿中的缺 陷进行钝化能够一定程度抑制离子迁移的发生. 如果能 够更加彻底地解决钙铁矿中离子迁移的问题, 那么就能 进一步提高钙钛矿 LED 器件的稳定性, 有效推进钻钛 矿 LED 器件实用化的进程.

\section{6 总结}

有机-无机杂化钙钛矿作为 LED 的发光层最大的优 势是荧光效率高、载流子迁移率高和制作成本低. 随着 近几年器件效率的飞速发展, 研究热情愈发高涨, 除了 需要进一步提高 LED 器件的效率, 器件的稳定性也是 实现其商业化应用的阻碍. 我们认为, 在今后的研究中, 可以将重点放在以下几个方面:

(1)红、蓝光的实现

钙铁矿 LED 中实现红、蓝光最大的问题是色光的 稳定性较差. 目前采用混合卤素的方法获得的红、蓝光 存在卤素相分离的问题, 色光的稳定性较差, 需要探索 能够抑制卤素相分离的方法, 可以从抑制表界面卤素离 子的迁移着手; 另外, 利用准二维钻钣矿的空间限域效 应实现发射峰的蓝移也是获得红、蓝光的可行途径, 但 是该方法需要保持准二维钲钛矿中不同 $n$ 值的相分布的 稳定性, 特别是通过大阳离子的设计来实现较小的相偏 析.

\section{(2)钙钛矿 LED 的高效率}

为了进一步提高器件效率, 除了选择合适的钝化分 子提高钻钛矿材料的 PLQY, 通过修饰界面提高载流子 注入的平衡, 还需要从出光的角度进行突破. 钙钛矿较 有机传输层具有更高的折射率, 使得部分光在发光层内 发生全反射无法发射出来. 因此, 在保持发光层较高的 PLQY 和载流子迁移率的前提下，可以通过对钙铁矿薄 膜的形貌、厚度及微纳结构的调控, 实现更高的出光效 率.

\section{(3)钙铁矿 LED 的高稳定性}

稳定性也是制约钙钣矿 LED 器件实现商业化应用 的重大阻碍, 其中电场下的离子迁移是器件性能衰减的 重要诱因. 因此, 在保证钙钠矿组分和相态稳定的前提 
下，需要抑制电场作用下的卤素离子迁移. 研究者可通 过钝化缺陷的策略来抑制缺陷诱导的卤素离子的迁移; 选择合适的钝化剂或添加剂保证钙钛矿发光层的迁移 率, 以降低器件工作过程中产生的热, 从而抑制热量激 发的缺陷的扩散和离子的迁移; 选择与卤素离子结合作 用较强的添加剂, 通过强化学相互作用将卤素离子钉扎 在晶体表界面, 抑制卤素空位的形成和卤素离子的迁 移.

\section{(4)非铅基钙钛矿}

铅基钻钛矿的毒性也是制约其应用发展的一个因 素, 因此以锡替代铅制备锡基钻钛矿光电器件受到广泛 关注. 锡基钙钛矿除了低毒的特点, 以锡为中心的八面 体形成的无机骨架的性质与铅基有很大不同. 在 $n=1$ 的铅基钙钛矿中, 激子结合能很大 (320 meV), 但是由 于激子热猝灭, 在室温下很难看到荧光 ${ }^{[29]}$; 而相应的 $n$ $=1$ 的锡基二维钲钛矿中, 测得激子结合能约 50 $\mathrm{meV}^{[57]}$, 远低于铅基二维钙钛矿的激子结合能 ${ }^{[115]}$; 并 且已有报道基于 $\mathrm{PEA}_{2} \mathrm{SnI}_{4}$ 二维钙钛矿的纯红光 $(632$ $\mathrm{nm})$ 的 LED 器件 EQE 已达到 5\%[116]. 因此, 锡基钙钛矿 的性质有很多独特的地方, 随着研究者们对锡基钙钛矿 性质的探索, 锡基钙钛矿 LED 器件的性能必将有很大 提升.

\section{作者简介}

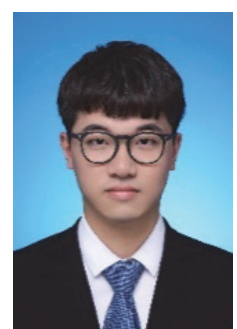

郭镇域, 北京大学工学院 2018 级硕士研究生, 研究方向 为有机无机杂化准二维钙钛矿发光二极管器件制备.

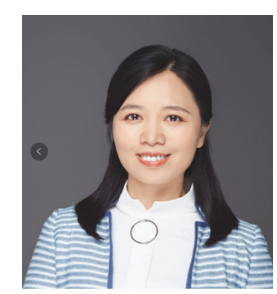

周欢萍, 2010 年博士毕业于北京大学化学与分子工程学 院. 2010 年至 2015 年期间, 于美国加州大学洛杉矶分校材料 科学与工程系从事博士后工作研究. 2015 年 7 月加入北京大学 工学院材料科学与工程系, 研究方向为新型光电半导体材料 以及相关器件.

\section{References}

[1] Kojima, A.; Teshima, K.; Shirai, Y.; Miyasaka, T. J. Am. Chem. Soc. 2009, 131, 6050

[2] Best Research-Cell Efficiencies (NREL, 2020), https://www.nrel. gov/pv/assets/pdfs/best-research-cell-efficiencies.20200925.pdf

[3] Ishihara, T.; Takahashi, J.; Goto, T. Phys. Rev. B 1990, 42, 11099.

[4] Tan, Z.-K.; Moghaddam, R. S.; Lai, M. L.; Docampo, P.; Higler, R.; Deschler, F.; Price, M.; Sadhanala, A.; Pazos, L. M.; Credgington, D. Nat. Nanotechnol. 2014, 9, 687.

[5] Xu, W.; Hu, Q.; Bai, S.; Bao, C.; Miao, Y.; Yuan, Z.; Borzda, T.; Barker, A. J.; Tyukalova, E.; Hu, Z.; Kawecki, M.; Wang, H.; Yan, Z.; Liu, X.; Shi, X.; Uvdal, K.; Fahlman, M.; Zhang, W.; Duchamp, M.; Liu, J.-M.; Petrozza, A.; Wang, J.; Liu, L.-M.; Huang, W.; Gao, F. Nat. Photonics. 2019, 13, 418.

[6] Lin, K.; Xing, J.; Quan, L. N.; de Arquer, F. P. G.; Gong, X.; Lu, J.; Xie, L.; Zhao, W.; Zhang, D.; Yan, C.; Li, W.; Liu, X.; Lu, Y.; Kirman, J.; Sargent, E. H.; Xiong, Q.; Wei, Z. Nature 2018, 562, 245.

[7] Dong, Y.; Wang, Y.-K.; Yuan, F.; Johnston, A.; Liu, Y.; Ma, D.; Choi, M.-J.; Chen, B.; Chekini, M.; Baek, S.-W.; Sagar, L. K.; Fan, J.; Hou, Y.; Wu, M.; Lee, S.; Sun, B.; Hoogland, S.; Quintero-Bermudez, R.; Ebe, H.; Todorovic, P.; Dinic, F.; Li, P.; Kung, H. T.; Saidaminov, M. I.; Kumacheva, E.; Spiecker, E.; Liao, L.-S.; Voznyy, O.; Lu, Z.-H.; Sargent, E. H. Nat. Nanotechnol. 2020, 15,668 .

[8] Wang, H.; Zhang, X.; Wu, Q.; Cao, F.; Yang, D.; Shang, Y.; Ning, Z.; Zhang, W.; Zheng, W.; Yan, Y.; Kershaw, S. V.; Zhang, L.; Rogach, A. L.; Yang, X. Nat. Commun. 2019, 10, 665.

[9] Shao, Y.; Xiao, Z.; Bi, C.; Yuan, Y.; Huang, J. Nat. Commun. 2014, $5,5784$.

[10] Li, N.; Tao, S.; Chen, Y.; Niu, X.; Onwudinanti, C. K.; Hu, C.; Qiu, Z.; Xu, Z.; Zheng, G.; Wang, L.; Zhang, Y.; Li, L.; Liu, H.; Lun, Y.; Hong, J.; Wang, X.; Liu, Y.; Xie, H.; Gao, Y.; Bai, Y.; Yang, S.; Brocks, G.; Chen, Q.; Zhou, H. Nat. Energy. 2019, 4, 408.

[11] Chen, Y.; Li, N.; Wang, L.; Li, L.; Xu, Z.; Jiao, H.; Liu, P.; Zhu, C.; Zai, H.; Sun, M.; Zou, W.; Zhang, S.; Xing, G.; Liu, X.; Wang, J.; Li, D.; Huang, B.; Chen, Q.; Zhou, H. Nat. Commun. 2019, 10, 1112.

[12] Tsai, H.; Nie, W.; Blancon, J.-C.; Stoumpos, C. C.; Asadpour, R.; Harutyunyan, B.; Neukirch, A. J.; Verduzco, R.; Crochet, J. J.; Tretiak, S. Nature 2016, 536, 312.

[13] Wang, J.; Wang, N.; Jin, Y.; Si, J.; Tan, Z. K.; Du, H.; Cheng, L.; Dai, X.; Bai, S.; He, H.; Ye, Z.; Lai, M. L.; Friend, R. H.; Huang, W. Adv. Mater. 2015, 27, 2311.

[14] Cao, Y.; Wang, N.; Tian, H.; Guo, J.; Wei, Y.; Chen, H.; Miao, Y.; Zou, W.; Pan, K.; He, Y.; Cao, H.; Ke, Y.; Xu, M.; Wang, Y.; Yang, M.; Du, K.; Fu, Z.; Kong, D.; Dai, D.; Jin, Y.; Li, G.; Li, H.; Peng, Q.; Wang, J.; Huang, W. Nature 2018, 562, 249.

[15] Deschler, F.; Price, M.; Pathak, S.; Klintberg, L. E.; Jarausch, D.-D.; Higler, R.; Hüttner, S.; Leijtens, T.; Stranks, S. D.; Snaith, H. J. J. Phys. Chem. Lett. 2014, 5, 1421.

[16] Sutherland, B. R.; Hoogland, S.; Adachi, M. M.; Wong, C. T.; Sargent, E. H. ACS Nano 2014, 8, 10947.

[17] Zhang, Q.; Ha, S. T.; Liu, X.; Sum, T. C.; Xiong, Q. Nano Lett. 2014, 14, 5995 .

[18] Chen, Q.; Wu, J.; Ou, X.; Huang, B.; Almutlaq, J.; Zhumekenov, A. A.; Guan, X.; Han, S.; Liang, L.; Yi, Z.; Li, J.; Xie, X.; Wang, Y.; Li, Y.; Fan, D.; Teh, D. B. L.; All, A. H.; Mohammed, O. F.; Bakr, O. M.; Wu, T.; Bettinelli, M.; Yang, H.; Huang, W.; Liu, X. Nature 2018, 561, 88 .

[19] Deng, W.; Jie, J.; Xu, X.; Xiao, Y.; Lu, B.; Zhang, X.; Zhang, X. Adv. Mater. 2020, 32, 1908340.

[20] Wei, Z.; Xing, J. J. Phys. Chem. Lett. 2019, 10, 3035.

[21] Kumawat, N. K.; Gupta, D.; Kabra, D. Energy Technol. 2017, 5, 1734.

[22] Wei, Z.; Xing, J. J. Phys. Chem. Lett. 2019, 10, 3035.

[23] He, Z.; Liu, Y.; Yang, Z.; Li, J.; Cui, J.; Chen, D.; Fang, Z.; He, H.; Ye, Z.; Zhu, H.; Wang, N.; Wang, J.; Jin, Y. ACS Photonics 2019, 6, 587.

[24] Matsushima, T.; Bencheikh, F.; Komino, T.; Leyden, M. R.; Sandanayaka, A. S. D.; Qin, C.; Adachi, C. Nature 2019, 572, 502.

[25] Yang, X. L.; Zhang, X. W.; Deng, J. X.; Chu, Z. M.; Jiang, Q.; Meng, J. H.; Wang, P. Y.; Zhang, L. Q.; Yin, Z. G.; You, J. B. Nat. Commun. 2018, 9, 8 .

[26] Manders, J. R.; Tsang, S. W.; Hartel, M. J.; Lai, T. H.; Chen, S.; Amb, C. M.; Reynolds, J. R.; So, F. Adv. Funct. Mater. 2013, 23, 2993.

[27] Yin, W. J.; Shi, T.; Yan, Y. Adv. Mater. 2014, 26, 4653.

[28] Xu, L.; Li, J.; Cai, B.; Song, J.; Zhang, F.; Fang, T.; Zeng, H. Nat. Commun. 2020, 11, 1 .

[29] Yuan, M. J.; Quan, L. N.; Comin, R.; Walters, G.; Sabatini, R.; 
Voznyy, O.; Hoogland, S.; Zhao, Y. B.; Beauregard, E. M.; Kanjanaboos, P.; Lu, Z. H.; Kim, D. H.; Sargent, E. H. Nat. Nanotechnol. 2016, 11, 872 .

[30] Wang, N.; Cheng, L.; Ge, R.; Zhang, S.; Miao, Y.; Zou, W.; Yi, C.; Sun, Y.; Cao, Y.; Yang, R. Nat. Photonics 2016, 10, 699.

[31] Xing, G.; Wu, B.; Wu, X.; Li, M.; Du, B.; Wei, Q.; Guo, J.; Yeow, E. K.; Sum, T. C.; Huang, W. Nat. Commun. 2017, 8, 14558.

[32] Xiao, Z. G.; Kerner, R. A.; Zhao, L. F.; Tran, N. L.; Lee, K. M.; Koh, T. W.; Scholes, G. D.; Rand, B. P. Nat. Photonics 2017, 11, 108.

[33] Cho, H.; Jeong, S.-H.; Park, M.-H.; Kim, Y.-H.; Wolf, C.; Lee, C.-L.; Heo, J. H.; Sadhanala, A.; Myoung, N.; Yoo, S. Science 2015, $350,1222$.

[34] Lee, J. W.; Choi, Y. J.; Yang, J. M.; Ham, S.; Jeon, S. K.; Lee, J. Y.; Song, Y. H.; Ji, E. K.; Yoon, D. H.; Seo, S.; Shin, H.; Han, G. S.; Jung, H. S.; Kim, D.; Park, N. G. ACS Nano 2017, 11, 3311.

[35] Jia, Y. H.; Neutzner, S.; Zhou, Y.; Yang, M.; Tapia, J. M. F.; Li, N.; Yu, H.; Cao, J.; Wang, J. P.; Petrozza, A.; Wong, C. P.; Zhao, N. Adv. Funct. Mater. 2020, 30, 1906875

[36] Yuan, Z.; Miao, Y.; Hu, Z.; Xu, W.; Kuang, C.; Pan, K.; Liu, P.; Lai, J.; Sun, B.; Wang, J.; Bai, S.; Gao, F. Nat. Commun. 2019, 10, 2818.

[37] Cho, H.; Wolf, C.; Kim, J. S.; Yun, H. J.; Bae, J. S.; Kim, H.; Heo, J. M.; Ahn, S.; Lee, T. W. Adv. Mater. 2017, 29, 1700579.

[38] Yantara, N.; Bhaumik, S.; Yan, F.; Sabba, D.; Dewi, H. A.; Mathews, N.; Boix, P. P.; Demir, H. V.; Mhaisalkar, S. J. Phys. Chem. Lett. 2015, 6,4360 .

[39] Wang, Z. B.; Luo, Z.; Zhao, C. Y.; Guo, Q.; Wang, Y. P.; Wang, F. Z.; Bian, X. M.; Alsaedi, A.; Hayat, T.; Tan, Z. A. J. Phys. Chem. C 2017, 121, 28132.

[40] Wang, J.; Song, C.; He, Z.; Mai, C.; Xie, G.; Mu, L.; Cun, Y.; Li, J.; Wang, J.; Peng, J.; Cao, Y. Adv. Mater. 2018, 30, 1804137.

[41] Miao, Y.; Ke, Y.; Wang, N.; Zou, W.; Xu, M.; Cao, Y.; Sun, Y.; Yang, R.; Wang, Y.; Tong, Y.; Xu, W.; Zhang, L.; Li, R.; Li, J.; He, H.; Jin, Y.; Gao, F.; Huang, W.; Wang, J. Nat. Commun. 2019, 10, 3624.

[42] Tsai, H.; Nie, W.; Blancon, J. C.; Stoumpos, C. C.; Asadpour, R.; Harutyunyan, B.; Neukirch, A. J.; Verduzco, R.; Crochet, J. J.; Tretiak, S.; Pedesseau, L.; Even, J.; Alam, M. A.; Gupta, G.; Lou, J.; Ajayan, P. M.; Bedzyk, M. J.; Kanatzidis, M. G. Nature 2016, 536, 312.

[43] Xing, J.; Zhao, Y.; Askerka, M.; Quan, L. N.; Gong, X.; Zhao, W.; Zhao, J.; Tan, H.; Long, G.; Gao, L.; Yang, Z.; Voznyy, O.; Tang, J.; Lu, Z. H.; Xiong, Q.; Sargent, E. H. Nat. Commun. 2018, 9, 3541.

[44] Quan, L. N.; Zhao, Y. B. A.; de Arquer, F. P. G.; Sabatini, R.; Walters, G.; Voznyy, O.; Comin, R.; Li, Y. Y.; Fan, J. Z.; Tan, H. R.; Pan, J.; Yuan, M. J.; Bakr, O. M.; Lu, Z. H.; Kim, D. H.; Sargent, E. H. Nano Lett. 2017, 17, 3701 .

[45] Zhou, N.; Shen, Y.; Li, L.; Tan, S.; Liu, N.; Zheng, G.; Chen, Q.; Zhou, H. J. Am. Chem. Soc. 2018, 140, 459.

[46] Yang, X.; Chu, Z.; Meng, J.; Yin, Z.; Zhang, X.; Deng, J.; You, J. J. Phys. Chem. Lett. 2019, 10, 2892.

[47] Chang, J.; Zhang, S.; Wang, N.; Sun, Y.; Wei, Y.; Li, R.; Yi, C.; Wang, J.; Huang, W. J. Am. Chem. Soc. 2018, 9, 881.

[48] Zou, W.; Li, R.; Zhang, S.; Liu, Y.; Wang, N.; Cao, Y.; Miao, Y.; Xu, M.; Guo, Q.; Di, D. Nat. Commun. 2018, 9, 1.

[49] Li, Z.; Chen, Z.; Yang, Y.; Xue, Q.; Yip, H.-L.; Cao, Y. Nat. Commun. 2019, 10, 1.

[50] Jiang, Y.; Qin, C.; Cui, M.; He, T.; Liu, K.; Huang, Y.; Luo, M.; Zhang, L.; Xu, H.; Li, S.; Wei, J.; Liu, Z.; Wang, H.; Kim, G. H.; Yuan, M.; Chen, J. Nat. Commun. 2019, 10, 1868.

[51] Liu, Y.; Cui, J.; Du, K.; Tian, H.; He, Z.; Zhou, Q.; Yang, Z.; Deng, Y.; Chen, D.; Zuo, X.; Ren, Y.; Wang, L.; Zhu, H.; Zhao, B.; Di, D.; Wang, J.; Friend, R. H.; Jin, Y. Nat. Photonics 2019, 13, 760.

[52] Yuan, S.; Wang, Z. K.; Xiao, L. X.; Zhang, C. F.; Yang, S. Y.; Chen, B. B.; Ge, H. T.; Tian, Q. S.; Jin, Y.; Liao, L. S. Adv. Mater. 2019, $31,1904319$.

[53] Yantara, N.; Jamaludin, N. F.; Febriansyah, B.; Giovanni, D.; Bruno, A.; Soci, C.; Sum, T. C.; Mhaisalkar, S.; Mathews, N. ACS Energy Lett. 2020, 5, 1593.

[54] Chu, Z.; Zhao, Y.; Ma, F.; Zhang, C. X.; Deng, H.; Gao, F.; Ye, Q.; Meng, J.; Yin, Z.; Zhang, X.; You, J. Nat. Commun. 2020, 11, 4165.

[55] Qin, C.; Matsushima, T.; Potscavage, W. J.; Sandanayaka, A. S. D.; Leyden, M. R.; Bencheikh, F.; Goushi, K.; Mathevet, F.; Heinrich, B.; Yumoto, G.; Kanemitsu, Y.; Adachi, C. Nat. Photonics 2019, 14, 70.

[56] Tsai, H.; Liu, C.; Kinigstein, E.; Li, M.; Tretiak, S.; Cotlet, M.; Ma, X.; Zhang, X.; Nie, W. Adv. Sci. 2020, 7, 1903202.
[57] Wang, Z.; Wang, F.; Zhao, B.; Qu, S.; Hayat, T.; Alsaedi, A.; Sui, L.; Yuan, K.; Zhang, J.; Wei, Z.; Tan, Z. a. J. Phys. Chem. Lett. 2020, 11,1120 .

[58] Yu, M.; Yi, C.; Wang, N.; Zhang, L.; Zou, R.; Tong, Y.; Chen, H.; Cao, Y.; He, Y.; Wang, Y.; Xu, M.; Liu, Y.; Jin, Y.; Huang, W.; Wang, J. Adv. Opt. Mater. 2018, 7, 1801575.

[59] Shang, Y.; Liao, Y.; Wei, Q.; Wang, Z.; Xiang, B.; Ke, Y.; Liu, W.; Ning, Z. Sci. Adv. 2019, 5, eaaw8072.

[60] Jin, Y.; Wang, Z. K.; Yuan, S.; Wang, Q.; Qin, C.; Wang, K. L.; Dong, C.; Li, M.; Liu, Y.; Liao, L. S. Adv. Funct. Mater. 2019, 30, 1908339.

[61] Protesescu, L.; Yakunin, S.; Bodnarchuk, M. I.; Krieg, F.; Caputo, R.; Hendon, C. H.; Yang, R. X.; Walsh, A.; Kovalenko, M. V. Nano Lett. 2015, 15, 3692.

[62] Zhang, F.; Zhong, H.; Chen, C.; Wu, X.-g.; Hu, X.; Huang, H.; Han, J.; Zou, B.; Dong, Y. ACS Nano 2015, 9, 4533.

[63] Wang, K. H.; Wu, L.; Li, L.; Yao, H. B.; Qian, H. S.; Yu, S. H Angew. Chem. Int. Ed. 2016, 128, 8468.

[64] Shamsi, J.; Rastogi, P.; Caligiuri, V.; Abdelhady, A. L.; Spirito, D.; Manna, L.; Krahne, R. ACS Nano 2017, 11, 10206.

[65] Shi, Y.; Xi, J.; Lei, T.; Yuan, F.; Dai, J.; Ran, C.; Dong, H.; Jiao, B.; Hou, X.; Wu, Z. ACS Appl. Mater. Interfaces 2018, 10, 9849.

[66] Mittal, M.; Jana, A.; Sarkar, S.; Mahadevan, P.; Sapra, S. J. Phys. Chem. Lett. 2016, 7, 3270.

[67] Chiba, T.; Hayashi, Y.; Ebe, H.; Hoshi, K.; Sato, J.; Sato, S.; Pu, Y.-J.; Ohisa, S.; Kido, J. Nat. Photonics 2018, 12, 681.

[68] Di Stasio, F.; Christodoulou, S.; Huo, N.; Konstantatos, G. Chem. Mater. 2017, 29, 7663.

[69] Liu, F.; Zhang, Y.; Ding, C.; Kobayashi, S.; Izuishi, T.; Nakazawa, N.; Toyoda, T.; Ohta, T.; Hayase, S.; Minemoto, T. ACS Nano 2017 $11,10373$.

[70] Yuan, X.; Hou, X.; Li, J.; Qu, C.; Zhang, W.; Zhao, J.; Li, H. Phys. Chem. Chem. Phys. 2017, 19, 8934.

[71] Li, Y.; Li, J.; Xu, L.; Chen, J.; Song, J. Acta Chim. Sinica 2020, doi: 10.6023/A20080386. (李严, 李金航，许蕾梦，陈嘉伟，宋继中， 化学学报, 2020, doi: 10.6023/A20080386.)

[72] Pan, J.; Quan, L. N.; Zhao, Y.; Peng, W.; Murali, B.; Sarmah, S. P.; Yuan, M.; Sinatra, L.; Alyami, N. M.; Liu, J.; Yassitepe, E.; Yang, Z.; Voznyy, O.; Comin, R.; Hedhili, M. N.; Mohammed, O. F.; Lu, Z. H.; Kim, D. H.; Sargent, E. H.; Bakr, O. M. Adv. Mater. 2016, 28, 8718 .

[73] Li, J.; Xu, L.; Wang, T.; Song, J.; Chen, J.; Xue, J.; Dong, Y.; Cai, B.; Shan, Q.; Han, B.; Zeng, H. Adv. Mater. 2017, 29, 1603885.

[74] Song, J.; Fang, T.; Li, J.; Xu, L.; Zhang, F.; Han, B.; Shan, Q.; Zeng, H. Adv. Mater. 2018, 30, 1805409.

[75] Yang, D.; Li, X.; Zhou, W.; Zhang, S.; Meng, C.; Wu, Y.; Wang, Y.; Zeng, H. Adv. Mater. 2019, 31, e1900767.

[76] Zhang, B. B.; Yuan, S.; Ma, J. P.; Zhou, Y.; Hou, J.; Chen, X.; Zheng, W.; Shen, H.; Wang, X. C.; Sun, B.; Bakr, O. M.; Liao, L. S.; Sun, H. T. J. Am. Chem. Soc. 2019, 141, 15423.

[77] Norris, D. J.; Yao, N.; Charnock, F. T.; Kennedy, T. A. Nano Lett. 2001, 1,3 .

[78] Pradhan, N.; Peng, X. J. Am. Chem. Soc. 2007, 129, 3339.

[79] Sahu, A.; Kang, M. S.; Kompch, A.; Notthoff, C.; Wills, A. W.; Deng, D.; Winterer, M.; Frisbie, C. D.; Norris, D. J. Nano Lett. 2012 $12,2587$.

[80] Zou, S.; Liu, Y.; Li, J.; Liu, C.; Feng, R.; Jiang, F.; Li, Y.; Song, J.; Zeng, H.; Hong, M.; Chen, X. J. Am. Chem. Soc. 2017, 139, 11443.

[81] Liu, H.; Wu, Z.; Shao, J.; Yao, D.; Gao, H.; Liu, Y.; Yu, W.; Zhang, H.; Yang, B. ACS Nano 2017, 11, 2239.

[82] Liu, W.; Lin, Q.; Li, H.; Wu, K.; Robel, I.; Pietryga, J. M.; Klimov, V. I. J. Am. Chem. Soc. 2016, 138, 14954.

[83] Parobek, D.; Roman, B. J.; Dong, Y.; Jin, H.; Lee, E.; Sheldon, M.; Son, D. H. Nano Lett. 2016, 16, 7376.

[84] Yao, J. S.; Ge, J.; Han, B. N.; Wang, K. H.; Yao, H. B.; Yu, H. L.; Li, J. H.; Zhu, B. S.; Song, J. Z.; Chen, C.; Zhang, Q.; Zeng, H. B.; Luo, Y.; Yu, S. H. J. Am. Chem. Soc. 2018, 140, 3626.

[85] Lu, M.; Zhang, X.; Bai, X.; Wu, H.; Shen, X.; Zhang, Y.; Zhang, W.; Zheng, W.; Song, H.; Yu, W. W.; Rogach, A. L. ACS Energy Lett. 2018, 3, 1571.

[86] Lu, M.; Zhang, X.; Zhang, Y.; Guo, J.; Shen, X.; Yu, W. W.; Rogach, A. L. Adv. Mater. 2018, 30, e1804691.

[87] Jiang, Q.; Zhao, Y.; Zhang, X.; Yang, X.; Chen, Y.; Chu, Z.; Ye, Q.; Li, X.; Yin, Z.; You, J. Nat. Photonics 2019, 13, 500

[88] Zheng, X.; Chen, B.; Dai, J.; Fang, Y.; Bai, Y.; Lin, Y.; Wei, H.; 
Zeng, X. C.; Huang, J. Nat. Energy. 2017, 2, 1.

[89] Abdi-Jalebi, M.; Andaji-Garmaroudi, Z.; Cacovich, S.; Stavrakas, C.; Philippe, B.; Richter, J. M.; Alsari, M.; Booker, E. P.; Hutter, E. M.; Pearson, A. J.; Lilliu, S.; Savenije, T. J.; Rensmo, H.; Divitini, G.; Ducati, C.; Friend, R. H.; Stranks, S. D. Nature 2018, 555, 497.

[90] deQuilettes, D. W.; Koch, S.; Burke, S.; Paranji, R. K.; Shropshire, A. J.; Ziffer, M. E.; Ginger, D. S. ACS Energy Lett. 2016, 1, 438.

[91] Wang, L.; McCleese, C.; Kovalsky, A.; Zhao, Y.; Burda, C. J. Am. Chem. Soc. 2014, 136, 12205.

[92] Braly, I. L.; deQuilettes, D. W.; Pazos-Outón, L. M.; Burke, S.; Ziffer, M. E.; Ginger, D. S.; Hillhouse, H. W. Nat. Photonics 2018, 12,355 .

[93] Noel, N. K.; Abate, A.; Stranks, S. D.; Parrott, E. S.; Burlakov, V. M.; Goriely, A.; Snaith, H. J. ACS Nano 2014, 8, 9815.

[94] Lin, Y.; Shen, L.; Dai, J.; Deng, Y.; Wu, Y.; Bai, Y.; Zheng, X.; Wang, J.; Fang, Y.; Wei, H.; Ma, W.; Zeng, X. C.; Zhan, X.; Huang, J. Adv. Mater. 2017, 29, 1604545.

[95] Wang, N.; Cheng, L.; Si, J.; Liang, X.; Jin, Y.; Wang, J.; Huang, W. Appl. Phys. Lett. 2016, 108, 141102.

[96] Ling, Y.; Tian, Y.; Wang, X.; Wang, J. C.; Knox, J. M.; Perez-Orive, F.; Du, Y.; Tan, L.; Hanson, K.; Ma, B.; Gao, H. Adv. Mater. 2016, $28,8983$.

[97] Lee, S.; Park, J. H.; Lee, B. R.; Jung, E. D.; Yu, J. C.; Di Nuzzo, D.; Friend, R. H.; Song, M. H. J. Phys. Chem. Lett. 2017, 8, 1784

[98] Wang, F.; Geng, W.; Zhou, Y.; Fang, H. H.; Tong, C. J.; Loi, M. A.; Liu, L. M.; Zhao, N. Adv. Mater. 2016, $28,9986$.

[99] Zhou, Y.; Wang, F.; Cao, Y.; Wang, J. P.; Fang, H. H.; Loi, M. A.; Zhao, N.; Wong, C. P. Adv. Energy Mater. 2017, 7, 1701048

[100] Quan, L.; Ma, D.; Zhao, Y.; Voznyy, O.; Yuan, H.; Bladt, E.; Pan, J.; García de Arquer, F. P.; Sabatini, R.; Piontkowski, Z.; Emwas, A.-H.; Todorović, P.; Quintero-Bermudez, R.; Walters, G.; Fan, J. Z.; Liu, M.; Tan, H.; Saidaminov, M. I.; Gao, L.; Li, Y.; Anjum, D. H.; Wei, N.; Tang, J.; McCamant, D. W.; Roeffaers, M. B. J.; Bals, S.; Hofkens, J.; Bakr, O. M.; Lu, Z.-H.; Sargent, E. H. Nat. Commun. 2020, 11,1 .
[101] Son, D. Y.; Kim, S. G.; Seo, J. Y.; Lee, S. H.; Shin, H.; Lee, D.; Park, N. G. J. Am. Chem. Soc. 2018, 140, 1358.

[102] Qiao, L.; Fang, W. H.; Long, R.; Prezhdo, O. V. Angew. Chem. Int Ed. 2019, 59, 4684

[103] Wu, T.; Li, J.; Zou, Y.; Xu, H.; Wen, K.; Wan, S.; Bai, S.; Song, T.; McLeod, J. A.; Duhm, S.; Gao, F.; Sun, B. Angew. Chem. Int. Ed. 2019, 59, 4099.

[104] Yang, J.-N.; Song, Y.; Yao, J.-S.; Wang, K.-H.; Wang, J.-J.; Zhu, B.-S.; Yao, M.-M.; Rahman, S. U.; Lan, Y.-F.; Fan, F.-J.; Yao, H.-B. J. Am. Chem. Soc. 2020, 59, 4099.

[105] Li, N.; Song, L.; Jia, Y.; Dong, Y.; Xie, F.; Wang, L.; Tao, S.; Zhao, N. Adv. Mater. 2020, 32, 1907786

[106] Yang, S.; Dai, J.; Yu, Z.; Shao, Y.; Zhou, Y.; Xiao, X.; Zeng, X. C.; Huang, J. J. Am. Chem. Soc. 2019, 141, 5781.

[107] Guo, Y.; Jia, Y.; Li, N.; Chen, M.; Hu, S.; Liu, C.; Zhao, N. Adv Funct. Mater. 2020, 30, 1910464.

[108] Fang, Z.; Chen, W.; Shi, Y.; Zhao, J.; Chu, S.; Zhang, J.; Xiao, Z. Adv. Funct. Mater. 2020, 30, 1909754

[109] Zhao, L.; Lee, K. M.; Roh, K.; Khan, S. U. Z.; Rand, B. P. Adv Mater. 2019, 31, 1805836.

[110] Zhang, X.; Lin, H.; Huang, H.; Reckmeier, C.; Zhang, Y.; Choy, W. C. H.; Rogach, A. L. Nano Lett. 2016, 16, 1415.

[111] Huang, H.; Lin, H.; Kershaw, S. V.; Susha, A. S.; Choy, W. C. H.; Rogach, A. L. J. Phys. Chem. Lett. 2016, 7, 4398.

[112] Zhang, L. Q.; Yang, X. L.; Jiang, Q.; Wang, P. Y.; Yin, Z. G.; Zhang, X. W.; Tan, H. R.; Yang, Y.; Wei, M. Y.; Sutherland, B. R.; Sargent, E. H.; You, J. B. Nat. Commun. 2017, 8, 8 .

[113] Ren, Z.; Xiao, X.; Ma, R.; Lin, H.; Wang, K.; Sun, X. W.; Choy, W. C. H. Adv. Funct. Mater. 2019, 29, 1905339.

[114] Ali, M. U.; Miao, J.; Cai, J.; Perepichka, D. F.; Yang, H.; Meng, H. ACS Appl. Mater. Interfaces 2020, 12, 18761.

[115] Zhou, N.; Huang, B.; Sun, M.; Zhang, Y.; Li, L.; Lun, Y.; Wang, X.; Hong, J.; Chen, Q.; Zhou, H. Adv. Energy Mater. 2019, 10, 1901566.

[116] Yuan, F.; Zheng, X.; Johnston, A.; Wang, Y.-K.; Zhou, C.; Dong, Y; Chen, B.; Chen, H.; Fan, J. Z.; Sharma, G. Sci. Adv. 2020, 6, eabb0253.

(Cheng, B.) 